\title{
Infinite paths in planar graphs IV, dividing cycles
}

\author{
Xingxing $\mathrm{Yu}^{*}$ \\ School of Mathematics \\ Georgia Institute of Technology \\ Atlanta, GA 30332, USA \\ and \\ Center for Combinatorics \\ Nankai University \\ Tianjin, 300071, China
}

February 10, 2006

\begin{abstract}
Nash-Williams conjectured that a 4-connected infinite planar graph contains a spanning 2-way infinite path if, and only if, the deletion of any finite set of vertices results in at most two infinite components. In this paper, we prove the Nash-Williams conjecture for graphs with no dividing cycles and for graphs with infinitely many vertex disjoint dividing cycles. A cycle in an infinite plane graph is called dividing if both regions of the plane bounded by this cycle contain infinitely many vertices of the graph.
\end{abstract}

*Partially supported by NSF grant DMS-0245530, NSA grant MDA-904-03-1-0052, and RGC grant HKU7056/04P 


\section{Introduction}

We use the terminology in [8], [9], and [10]. For convenience we repeat some here. Let $H$ be a (finite or infinite) subgraph of a (finite or infinite) graph $G$, let $v_{1}, \ldots, v_{k} \in V(G)$, and $\left\{u_{i}, w_{i}\right\} \subseteq V(H) \cup\left\{v_{1}, \ldots, v_{k}\right\}, i=1, \ldots, m$. Then $H+\left\{v_{1}, \ldots, v_{k}, u_{1} w_{1}, \ldots, u_{m} w_{m}\right\}$ denotes the graph with vertex set $V(H) \cup\left\{v_{1}, \ldots, v_{k}\right\}$ and edge set $E(H) \cup\left\{u_{1} w_{1}, \ldots, u_{k} w_{k}\right\}$. For any $x \in V(H) \cup E(H)$, we write $H+x$ instead of $H+\{x\}$.

Let $C$ be a cycle in a plane graph $G$ and let $x, y \in V(C)$. When $x \neq y$ then $x C y$ denotes the subpath of $C$ from $x$ to $y$ in clockwise order, and when $x=y$ then $x C y$ denotes the trivial path consisting of $x=y$ only. For a (finite or infinite) path $P$ and $x, y \in V(P)$, we use $x P y$ to denote the unique finite path in $P$ between $x$ and $y$.

By the Jordan curve theorem, each cycle $C$ in a (finite or infinite) plane graph $G$ divides the plane into two closed regions whose intersection is $C$. If $G$ is infinite and exactly one of these two closed regions, say $\mathcal{D}$, contains a finite subgraph of $G$, then we use $I_{G}(C)$ to denote the subgraph of $G$ contained in $\mathcal{D}$. If there is no danger of confusion, we use $I(C)$ instead of $I_{G}(C)$. Note that $C \subseteq I(C)$, and if $I(C)=C$ then $C$ is a facial cycle.

A graph $G$ is $k$-indivisible, where $k$ is a positive integer, if, for every finite $X \subseteq V(G)$, $G-X$ has at most $k-1$ infinite components. Nash-Williams ([2], [3], and [7]) conjectured that a 4 -connected infinite planar graph contains a spanning 2-way infinite path if, and only if, $G$ is 3-indivisible.

In [8] and [9], the Nash-Williams conjecture is established for 2-indivisible graphs. To deal with those graphs which are 3 -indivisible but not 2-indivisible, we define dividing cycles in an infinite plane graph $G$ as those cycles $C$ for which $I_{G}(C)$ is not defined. A non-dividing cycle in $G$ is then a cycle which is not dividing. Let $\gamma(G)$ denote the maximum number of vertex disjoint dividing cycles in an infinite plane graph $G$. With this notation, we may divide 3 -indivisible infinite plane graphs $G$ into three classes: those with $\gamma(G)=0$ (including all 2-indivisible graphs), those with $\gamma(G)=\infty$, and those for which $\gamma(G)$ is a positive integer. (Note that, when $\gamma(G)=0$, the drawing of $G$ may be modified to give a VAP-free drawing of $G$; see [5] and [1].) The objective of this paper is to give a proof of the following result, which establishes the Nash-Williams conjecture for two of these three classes.

(1.1) Theorem. Let $G$ be a 4-connected 3-indivisible infinite plane graph, and assume that $\gamma(G)=0$ or $\gamma(G)=\infty$. Then $G$ contains a spanning 2-way infinite path.

Throughout the rest of the paper, graphs will be finite unless it is clear from the context or otherwise mentioned. In Section 2 we summarize those concepts and results from [8], [9] and [10] which will be used in this paper. We prove in Section 3 three lemmas 
concerning 2-way infinite Tutte paths in two special classes of graphs. These lemmas will serve as bases for inductive arguments. Section 4 includes results which show that certain finite sequences of non-dividing cycles guarantee the existence of a 2-way infinite Tutte path. Theorem (1.1) will be proved in Section 5 for graphs with $\gamma(G)=0$. The proof of Theorem (1.1) will then be completed in Section 6 .

\section{Nets and Tutte paths}

A net in an infinite plane graph $G$ is a sequence $N:=\left(C_{1}, C_{2}, \ldots\right)$ of cycles in $G$ such that $I\left(C_{i}\right)$ is defined for all $i \geq 1$, and the following properties are satisfied:

(1) $I\left(C_{i}\right) \subseteq I\left(C_{i+1}\right)$ for all $i \geq 1$,

(2) $\bigcup_{i=1}^{\infty} I\left(C_{i}\right)=G$, and

(3) either $C_{i} \cap C_{j}=\emptyset$ for all $i \neq j$, or for $i \geq 1, C_{i} \cap C_{i+1}$ is a non-trivial path, $C_{i} \cap C_{i+1} \subseteq C_{i+1} \cap C_{i+2}$, and neither endvertex of $C_{i} \cap C_{i+1}$ is an endvertex of $C_{i+1} \cap C_{i+2}$.

If $C_{i} \cap C_{j}=\emptyset$ for all $i \neq j$, then $N$ is called a radial net; otherwise, $N$ is a ladder net. Let $\partial N=\emptyset$ if $N$ is a radial net; otherwise, let $\partial N=\bigcup_{i=1}^{\infty}\left(C_{i} \cap C_{i+1}\right)$.

Let $G$ be a (finite or infinite) graph and $H$ be a (finite or infinite) subgraph of $G$. An $H$-bridge of $G$ is a (finite or infinite) subgraph of $G$ which is induced by either (1) an edge of $E(G)-E(H)$ whose incident vertices are in $V(H)$ or (2) the edges contained in a component of $G-V(H)$ and the edges from that component to $H$. Also, we say that $G$ is $(4, H)$-connected if, for any $T \subseteq V(G)$ with $|T| \leq 3$, every component of $G-T$ contains a vertex of $H$. The following result is Theorem (2.1) in [10] (its 4-connected version is shown in [9]), which gives a structural description of graphs with nets.

(2.1) Theorem. Let $G$ be a 2-connected 2-indivisible infinite plane graph with a facial cycle $C$ such that $G$ is $(4, C)$-connected, and let $S$ denote the set of vertices of $G$ of infinite degree. Then $|S| \leq 2$, and there is a set $F$ of edges of $G$ such that

(1) for any $f \in F, f$ is incident with a vertex in $S$,

(2) $G-F$ has a net $N=\left(C_{1}, C_{2}, \ldots\right), C \subseteq I\left(C_{1}\right), S \subseteq \partial N$, and for any $f \in F$ both incident vertices of $f$ are contained in a common infinite $S$-bridge of $\partial N$,

(3) if $|S|=1$, then either one $S$-bridge of $\partial N$ contains all vertices incident with edges in $F$ or each $S$-bridge of $\partial N$ contains infinitely many vertices incident with edges in $F$, and 
(4) if $|S|=2$, then for any $T \subseteq V(G)-S$ with $|T| \leq 3, S$ is contained in a component of $(G-F)-T$.

For an infinite plane graph $G$, let $\partial G$ denote the subgraph of $G$ such that for each $x \in V(G) \cup E(G), x \in \partial G$ if and only if $x \notin(E(I(D))-E(D)) \cup(V(I(D))-V(D))$ for every cycle $D$ in $G$. Clearly, $\partial G=\emptyset$ when $G$ admits a radial net. From Theorem (2.1), we can show that when $G$ does not admit a radial net then $\partial G$ is a path, or a 1-way infinite path, or a 2-way infinite path. The following observation will be useful.

(2.2) Lemma. Let $G$ be a 2-connected infinite plane graph and $C$ be a facial cycle of $G$ such that $G$ is $(4, C)$-connected. If $G$ is 2-indivisible, then all but one face of $G$ are bounded by cycles, and $\partial G$ is precisely the subgraph of $G$ lies on the boundary of the exceptional face of $G$.

Proof. Suppose $G$ is 2-indivisible and let $R$ be a face of $G$. Suppose $R$ is incident with a vertex or edge which is not in $\partial G$. Then there exists some cycle $D$ in $G$ such that $R$ is incident with some element of $(E(I(D))-E(D)) \cup(V(I(D))-V(D))$. This shows that $R$ is a face of $I(D)$. Since $I(D)$ is a 2-connected plane graph, $R$ is bounded by a cycle. Now assume that all vertices or edges of $G$ incident with $R$ are in $\partial G$. Then since $G$ is 2-indivisible, it follows from Theorem (2.1) that $\partial G$ is precisely the subgraph of $G$ that lies on the boundary of $R$.

The next result is a generalization of Lemma (2.3) in [9].

(2.3) Lemma. Let $G$ be a 2-connected infinite plane graph and let $C$ be a facial cycle of $G$ such that $G$ is $(4, C)$-connected and $\gamma(G)=0$. Then there is an infinite sequence $\left(D_{1}, D_{2}, \ldots\right)$ of cycles in $G$ such that $C \subseteq I\left(D_{1}\right)$ and the following properties hold:

(1) for each $i \geq 1, I\left(D_{i}\right) \subseteq I\left(D_{i+1}\right)$, and $D_{i} \cap D_{i+1}$ is minimal among all subgraphs $D_{i} \cap D^{*}$ arising from cycles $D^{*}$ in $G$ such that $I\left(D_{i}\right) \subseteq I\left(D^{*}\right)$,

(2) for each $i \geq 1, G$ has no finite $I\left(D_{i}\right)$-bridge,

(3) for each $i \geq 1, D_{i} \cap D_{i+1} \subseteq D_{i+1} \cap D_{i+2}$, and

(4) $\bigcup_{i \geq 1} I\left(D_{i}\right)=G$.

The proof of Lemma (2.3) in [9] uses two properties: (a) for any finite $X \subseteq V(G)$, $G-X$ has only finitely many components, and (b) every cycle in $G$ is non-dividing (implied by cohesiveness). In the above lemma, (a) is guaranteed by the assumption that $G$ is planar and $(4, C)$-connected, and (b) is guaranteed by the assumption that $\gamma(G)=0$. 
In the remainder of this section, we state several results concerning Tutte paths in finite or infinite plane graphs. Let $G$ be a (finite or infinite) graph and $H$ be a (finite or infinite) subgraph of $G$. If $B$ is an $H$-bridge of $G$, then the vertices in $V(H \cap B)$ are called attachments of $B$ (on $H$ ). The subgraph $H$ is a Tutte subgraph of $G$ if every $H$-bridge of $G$ is finite and has at most three attachments. For a (finite or infinite) subgraph $C$ of $G$, we say that $H$ is a $C$-Tutte subgraph of $G$ if $H$ is a Tutte subgraph of $G$ and every $H$-bridge of $G$ containing an edge of $C$ has at most two attachments. A (finite or infinite) Tutte path is a (finite or infinite) path which is a Tutte subgraph.

The following result is the main theorem in [7].

(2.4) Lemma. Let $G$ be a 2-connected plane graph with a facial cycle $C$. Assume that $x \in V(C), e \in E(C)$, and $y \in V(G-x)$. Then $G$ contains a $C$-Tutte path $P$ from $x$ to $y$ such that $e \in E(P)$.

The next result is (2.6) from [4].

(2.5) Lemma. Let $G$ be a 2-connected plane graph with a facial cycle $C$. Let $u, v \in$ $V(C)$ be distinct, let $e, f \in E(C)$, and assume that $u, v, e, f$ occur on $C$ in clockwise order. Then $G$ contains a $v C u$-Tutte path $P$ from $u$ to $v$ such that $\{e, f\} \subseteq E(P)$.

We remark here that both Lemma (2.4) and Lemma (2.5) may be applied when $e$ or $f$ or both are vertices. We need Lemma (3.3) from [10], which will be convenient for extending Tutte paths.

(2.6) Lemma. Let $K$ be a connected (finite or infinite) plane graph, $C$ be a facial walk of $K, Q$ be a path between $p$ and $q$ on $C, u \in V(C)-V(Q), L$ be a subgraph of $K-V(Q)$, and $Q^{\prime}$ be a cycle in $L$ or a path in $L$ or a 2-way infinite path in $L$. Suppose the following three conditions are satisfied:

(1) for any $(L \cup Q)$-bridge $B$ of $K,|V(B \cap L)| \leq 1$ and $V(B \cap L) \subseteq V\left(Q^{\prime}\right)$,

(2) $K-V(L)$ is finite and all vertices of $K-V(L)$ have finite degree in $K$, and

(3) $L$ contains a $Q^{\prime}$-Tutte subgraph $T$ with $u \in V(T)$ and $\left|V\left(Q^{\prime}\right) \cap V(T)\right| \geq 2$.

Then $K-V(T)$ contains a path $S$ between $p$ and $q$ such that $S \cup T$ is a $Q$-Tutte subgraph of $K$, and every $T$-bridge of $L$ containing no edge of $Q^{\prime}$ is also an $(S \cup T)$-bridge of $K$.

The following result is Corollary (3.7) in [9].

(2.7) Lemma. Let $G$ be a 2-connected infinite plane graph with a ladder net $N$, and let $x \in V(\partial N)$ and $u v \in E(\partial N)$ such that $u \in V(x \partial N v)$. Then $G$ contains a 1-way infinite $\partial N$-Tutte path $P$ from $x$ such that $u v \in E(P)$ and $u \in V(x P v)$. 
We also need Theorem (1.2) from [10].

(2.8) Theorem. Let $G$ be a 2-connected 2-indivisible infinite plane graph, let $C$ be a facial cycle of $G$, let $x \in V(C)$ and $u v \in E(C)$ with $x \neq v$, and let $Q$ denote the subpath of $C-v$ between $u$ and $x$. Assume that $G$ is $(4, C)$-connected and $v$ is contained in the infinite component of $G-V(Q)$. Then $G$ contains a 1-way infinite $C$-Tutte path $P$ from $x$ such that $u v \in E(P)$ and $u \in V(x P v)$.

\section{Two-way infinite Tutte paths}

The goal of this section is to prove three results on 2-way infinite Tutte paths. These results will be used as bases for inductive arguments.

(3.1) Lemma. Let $G$ be a 2-connected 3-indivisible infinite plane graph, let $C$ be a facial cycle of $G$, and let $u, v \in V(C)$ be distinct such that $G$ is $(4, C)$-connected and $G-\{u, v\}$ has two infinite components. Then for any $e \in E(C), G$ contains a 2-way infinite $C$-Tutte path through $e$.

Proof. Without loss of generality, we may assume that the face of $G$ bounded by $C$ is an open disc. Since $G$ is $(4, C)$-connected, $G$ has at most three $\{u, v\}$-bridges: $G_{1}$ containing $v C u, G_{2}$ containing $u C v$, and possibly a third $\{u, v\}$-bridge induced by $u v$ (when $u v \in E(G)$ ). Since $G-\{u, v\}$ has two infinite components, $G_{1}$ and $G_{2}$ are infinite. For each $i \in\{1,2\}$, let $B_{i}$ be the infinite block of $G_{i}$. Since $G$ is 2-connected, $B_{1} \cap v C u$ and $B_{2} \cap u C v$ are nontrivial paths. Let $u^{\prime} \in V\left(B_{2} \cap u C v\right)$ with $u C u^{\prime}$ minimal, and let $v^{\prime} \in V\left(B_{1} \cap v C u\right)$ with $v C v^{\prime}$ minimal. See Figure 1, where the possible edge $u v$ is not drawn. Let $G^{\prime}:=G$ if $u v \notin E(G)$; otherwise, let $G^{\prime}:=G-u v$. Then $G^{\prime}$ has exactly two infinite $\left\{u^{\prime}, v^{\prime}\right\}$-bridges, one containing $B_{1}$ and the other containing $B_{2}$.
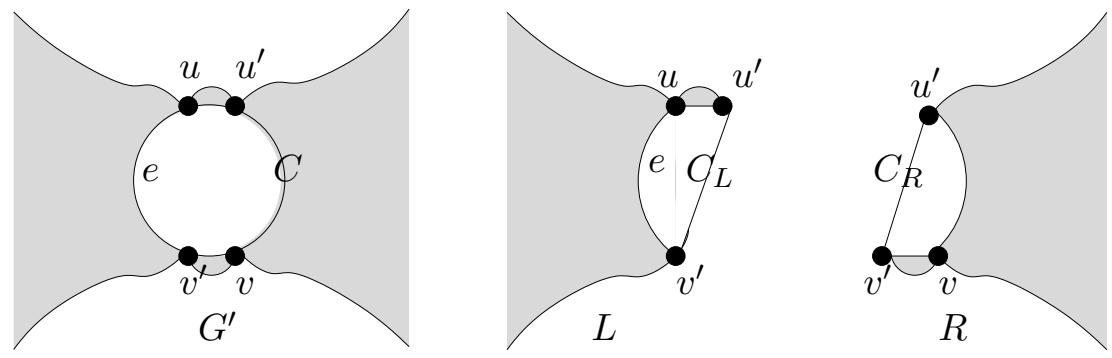

Figure 1: $G, L$ and $R$

Since $G$ is $(4, C)$-connected, neither $u^{\prime} C v$ nor $v^{\prime} C u$ is an edge; for otherwise, $G-$ $\left\{u^{\prime}, v\right\}$ or $G-\left\{u, v^{\prime}\right\}$ has a component containing no vertex of $C$, a contradiction. Let $L$ 
be obtained from $G^{\prime}$ by replacing the $\left\{u^{\prime}, v^{\prime}\right\}$-bridge of $G^{\prime}$ containing $B_{2}$ with the edge $u^{\prime} v^{\prime}$, and let $R$ be obtained from $G^{\prime}$ by replacing the $\left\{u^{\prime}, v^{\prime}\right\}$-bridge of $G^{\prime}$ containing $B_{1}$ with the edge $v^{\prime} u^{\prime}$. Let $C_{L}:=v^{\prime} C u^{\prime}+u^{\prime} v^{\prime}$ and $C_{R}:=u^{\prime} C v^{\prime}+v^{\prime} u^{\prime}$. We may assume that the edges are added so that the faces of $L$ and $R$ bounded by $C_{L}$ and $C_{R}$, respectively, are open discs. See Figure 1. Because $G$ is 3-indivisible, both $L$ and $R$ are 2-indivisible. Since $G$ is $(4, C)$-connected and since $V\left(C_{L}\right) \cup V\left(C_{R}\right)=V(C), L$ must be $\left(4, C_{L}\right)$-connected and $R$ must be $\left(4, C_{R}\right)$-connected.

By symmetry, we may assume that $e \in E\left(v^{\prime} C u^{\prime}\right)$, and let $e=a b$ so that $v^{\prime}, b, a, u^{\prime}$ occur on $C_{L}$ in clockwise order. Since $v^{\prime} \in B_{1}$, we see that $b$ is in the infinite component of $L-V\left(a C_{L} u^{\prime}\right)$. Hence, by Theorem (2.8), there is a 1-way infinite $C_{L}$-Tutte path $P_{L}$ from $u^{\prime}$ in $L$ such that $e \in E\left(P_{L}\right)$ and $a \in V\left(u^{\prime} P_{L} b\right)$. By planarity, $u^{\prime} v^{\prime} \notin E\left(P_{L}\right)$ and, therefore, $u \in V\left(P_{L}\right)$. We claim that $v^{\prime} \in V\left(P_{L}\right)$. For otherwise $v^{\prime}$ is contained in a $P_{L}$-bridge $B$ of $L$. Clearly, $u^{\prime} \in V\left(B \cap P_{L}\right)$. Since $P_{L}$ is a $C_{L}$-Tutte path of $L$, $\left|V\left(B \cap P_{L}\right)\right|=2$ and $B$ is finite. Let $v^{\prime \prime} \in V\left(B \cap P_{L}\right)-\left\{u^{\prime}\right\}$. Then $v^{\prime \prime}$ lies on $C$ and $v^{\prime} C v^{\prime \prime}-v^{\prime \prime} \nsubseteq B_{1}$, contradicting the choice of $v^{\prime}$.

If $v=v^{\prime}$ or $u v \notin E(G)$ then we use Theorem (2.8) to find a 1-way infinite $C_{R^{-}}$-Tutte path $P_{R}$ in $R$ from $v^{\prime}$ and through $v^{\prime} u^{\prime}$. It is easy to see that $P:=P_{L} \cup\left(P_{R}-v^{\prime}\right)$ is a 2-way infinite $C$-Tutte path in $G$ such that $e \in E(P)$.

Now assume $v \neq v^{\prime}$ and $u v \in E(G)$. Suppose $u=u^{\prime}$. In $R$ we use Theorem (2.8) to find a 1-way infinite $C_{R}$-Tutte path $P_{R}$ from $u^{\prime}$ and through $u^{\prime} v^{\prime}$. Then $v \in V\left(P_{R}\right)$. Let $P_{u}$ denote the infinite $u$-bridge of $P_{L}$ and $P_{v}$ denote the infinite $v$-bridge of $P_{R}$. Clearly $e \in E\left(P_{u}\right)$. It is easy to verify that $P:=\left(P_{u} \cup P_{v}\right)+u v$ gives the desired 2-way infinite $C$-Tutte path in $G$.

Hence we may assume $u \neq u^{\prime}$. Suppose $e \in E(v C u)$. In $R$, we use Theorem (2.8) to find a 1-way infinite $C_{R}$-Tutte path $P_{R}$ from $v^{\prime}$ and through the edge of $u^{\prime} C v^{\prime}$ incident with $v^{\prime}$. Then $v^{\prime} u^{\prime} \notin E\left(P_{R}\right)$ and $v \in V\left(P_{R}\right)$. By a similar argument as above for showing $v^{\prime} \in V\left(P_{L}\right)$, we can show that $u^{\prime} \in V\left(P_{R}\right)$. Let $P_{u}$ denote the infinite $u$-bridge of $P_{L}$ and $P_{v}$ denote the infinite $v$-bridge of $P_{R}$. Since $e \in E(v C u), e \in E\left(P_{u}\right)$. It is easy to verify that $P:=\left(P_{u} \cup P_{v}\right)+u v$ gives the desired 2-way infinite $C$-Tutte path in $G$.

To deal with the remaining case when $e \in E\left(u C u^{\prime}\right)$, we view $G_{i}+u v$ (for each $1 \leq$ $i \leq 2$ ) as a plane graph with a facial cycle $C_{i}$, where $C_{1}=v C u+u v$ and $C_{2}=u C v+u v$. In $G_{1}+u v$ we apply Theorem (2.8) to find a 1 -way infinite $C_{1}$-Tutte path $P_{1}$ from $u$ through $u v$. In $G_{2}+u v$ we apply Theorem (2.8) to find a 1-way infinite $C_{2}$-Tutte path $P_{2}$ from $v$ through $e$. Because $\left\{v, u^{\prime}\right\}$ is a 2 -cut in $G_{2}$, we see that $v u \in E\left(P_{2}\right)$. Hence, $P:=P_{1} \cup P_{2}$ gives the desired 2-way infinite $C$-Tutte path in $G$.

For the next two lemmas, we need additional notation. Let $G$ be a 2-connected 3 -indivisible infinite plane graph, and let $C$ be a facial cycle of $G$ such that $G$ is $(4, C)$ connected. Let $H$ be an infinite block of $G-V(C)$ and let $D$ be the cycle of $H$ which bounds the face of $H$ containing $C$. See Figure 2. Let $w_{1}, \ldots, w_{b}$ denote the attachments 


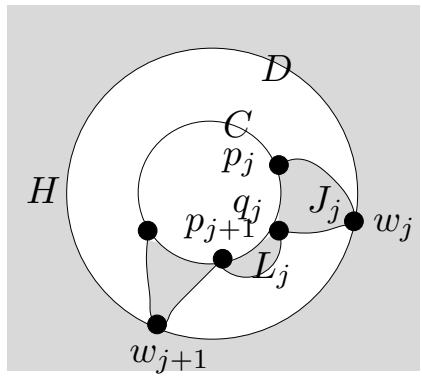

Figure 2: Illustration for Lemma (3.2)

on $H$ of $(H \cup C)$-bridges of $G$ which occur on $D$ in clockwise order. Let $p_{j}, q_{j} \in V(C)$ with $p_{j} C q_{j}$ maximal such that $\left\{p_{j}, w_{j}\right\}$ is contained in an $(H \cup C)$-bridge of $G,\left\{q_{j}, w_{j}\right\}$ is contained in an $(H \cup C)$-bridge of $G$, and any $(H \cup C)$-bridge of $G$ containing some $w_{l} \neq w_{j}$ contains no vertex of $V\left(p_{j} C q_{j}\right)-\left\{p_{j}, q_{j}\right\}$. Note that $p_{j}$ and $q_{j}$ are well defined because $G$ is $(4, C)$-connected. Let $J_{j}$ denote the union of $p_{j} C q_{j}$ and those $(H \cup C)$ bridges of $G$ whose attachments are all contained in $V\left(p_{j} C q_{j}\right) \cup\left\{w_{j}\right\}$. (Note that if $p_{j}=q_{j}$ then $J_{j}$ is induced by a single edge.) Let $L_{j}$ denote the union of $q_{j} C p_{j+1}$ and those $(H \cup C)$-bridges of $G$ whose attachments are all contained in $V\left(q_{j} C p_{j+1}\right)$, where $p_{b+1}=p_{1}$.

(3.2) Lemma. Let $G, C, H, D$ and $w_{j}, J_{j}, L_{j}(1 \leq j \leq b)$ be defined as above. Let $e \in E(C)$. Suppose there is some $1 \leq j \leq b$ such that $L_{j}$ is infinite and $e \notin E\left(L_{j}\right)$. Then $G$ contains a 2-way infinite $C$-Tutte path through $e$.

Proof. Without loss of generality, we may assume that $j=1$. Since $e \in E\left(p_{2} C q_{1}\right)$, $e \in E\left(p_{r} C p_{r+1}\right)$ for some $w_{r} \neq w_{1}$ or $e \in E\left(q_{r-1} C q_{r}\right)$ for some $w_{r} \neq w_{2}$. Note the symmetry between clockwise and counter clockwise orientations of $C$, and also note the symmetry between $w_{1}$ and $w_{2}$. We may therefore assume that $e \in E\left(p_{r} C p_{r+1}\right)$ for some $w_{r} \neq w_{1}$.

Since $G$ is 3 -indivisible and $L_{1}$ is infinite, $H$ is 2-indivisible. Since $G$ is $(4, C)$ connected and by planarity, $H$ is $(4, D)$-connected. Hence by Theorem $(2.8), H$ contains a 1-way infinite $D$-Tutte path $P$ from $w_{1}$ and through $w_{r}$.

Since $G$ is 2-connected and $L_{1}$ is infinite, $L_{1}-q_{1}$ contains a 1 -way infinite path from $p_{2}$. Let $L_{1}^{\prime}:=L_{1}+q_{1} p_{2}$ such that $C_{1}^{\prime}:=q_{1} C p_{2}+q_{1} p_{2}$ is a facial cycle of $L_{1}^{\prime}$. By Theorem (2.8), $L_{1}^{\prime}$ has a 1-way infinite $C_{1}^{\prime}$-Tutte path $Q_{1}$ from $q_{1}$ such that $q_{1} p_{2} \in E\left(Q_{1}\right)$.

In $J_{1}+p_{1} w_{1}$, we apply Lemma (2.4) to find a $p_{1} C q_{1}$-Tutte path $P_{1}$ from $w_{1}$ to $p_{1}$ and through $q_{1}$. 
We apply Lemma (2.6) to $K:=G-V\left(\left(J_{1} \cup L_{1}\right)-\left\{p_{1}, p_{2}, w_{1}\right\}\right)$, $H, p_{2} C p_{1}, D, P, p_{2}, p_{1}, w_{1}$ (as $K, L, Q, Q^{\prime}, T, p, q, u$, respectively). Note that the conditions of Lemma (2.6) are satisfied. In particular, $w_{1}, w_{r} \in V(P)$ implies that $|V(P \cap D)| \geq 2$. Hence, by Lemma (2.6), there is a path $S$ in $K-V(P)$ between $p_{2}$ and $p_{1}$ such that $S \cup P$ is a $p_{2} C p_{1}$-Tutte subgraph in $K$ and every $P$-bridge of $H$ containing no edge of $D$ is also an $(S \cup P)$-bridge of $K$.

We may assume that $e \in E(S)$. This may be seen as follows. By planarity and because $w_{r} \in V(P), p_{r}$ and $p_{r+1}$ are cut vertices of $K-V(P)$. Hence, $p_{r}, p_{r+1} \in V(S)$, $\left(J_{r} \cup L_{r}\right)-w_{r}$ is a $\left\{p_{r}, p_{r+1}\right\}$-bridge of $K-V(P)$, and $p_{r} S p_{r+1} \subseteq\left(J_{r} \cup L_{r}\right)-w_{r}$. In $\left(J_{r} \cup L_{r}\right)+p_{r+1} w_{r}$, we apply Lemma $(2.5)$ to find a $p_{r} C p_{r+1}$-Tutte path $S_{r}$ from $p_{r}$ to $w_{r}$ such that $p_{r+1} w_{r}, e \in E\left(S_{r}\right)$. By replacing the subpath $p_{r} S p_{r+1}$ of $S$ with $S_{r}-w_{r}$, we obtain the desired path $S$ through $e$.

Now $P^{*}:=P \cup P_{1} \cup S \cup\left(Q_{1}-q_{1}\right)$ is a 2-way infinite path through $e$. Note that every $P^{*}$-bridge of $G$ is one of the following: an $(S \cup P)$-bridge of $H$, or a $P_{1}$-bridge of $J_{1}$, or a $Q_{1}$-bridge of $L_{1}^{\prime}$. Hence, $P^{*}$ is a 2 -way infinite $C$-Tutte path in $G$ through $e$.

(3.3) Lemma. Let $G, C, H, D$ and $w_{j}, J_{j}, L_{j}(1 \leq j \leq b)$ be defined as above. Let $e \in E(C)$. Suppose there is some $1 \leq j \leq b$ such that $J_{j}$ is infinite and $e$ is not contained in the unique infinite block of $J_{j}-w_{j}$. Then $G$ contains a 2-way infinite $C$-Tutte path through $e$.

Proof. Without loss of generality, we may assume that $j=1$. If $e \in E\left(J_{1}\right)$, we choose an arbitrary $w_{r} \neq w_{1}$. Since $e$ is not contained in the infinite block of $J_{j}-w_{j}$, there is a vertex $v \in V\left(p_{1} C q_{1}-\left\{p_{1}, q_{1}\right\}\right)$ such that the infinite $\left\{w_{1}, v\right\}$-bridge of $J_{1}$ does not contain $e_{1}$. In this case, let $J_{1}^{*}$ denote the infinite $\left\{w_{1}, v\right\}$-bridge of $J_{1}$. Now assume $e \notin E\left(J_{1}\right)$. Then $e \in E\left(q_{1} C p_{1}\right)$, and hence $e \in E\left(p_{r} C p_{r+1}\right)$ for some $w_{r} \neq w_{1}$ or $e \in E\left(q_{r-1} C q_{r}\right)$ for some $w_{r} \neq w_{1}$. Let $J_{1}^{*}=J_{1}$ and $v:=p_{1}$.

Note the symmetry between clockwise and counter clockwise orientations of $C$. We may therefore assume that when $e \notin E\left(J_{1}\right)$ we have $e \in E\left(p_{r} C p_{r+1}\right)$ for some $w_{r} \neq w_{1}$, and when $e \in E\left(J_{1}\right)$ then $q_{1} \in V\left(J_{1}^{*}\right)$.

Since $G$ is 3 -indivisible and $J_{1}$ is infinite, $H$ is 2-indivisible. Since $G$ is $(4, C)$ connected and by planarity, $H$ is $(4, D)$-connected. Hence by Theorem $(2.8), H$ contains a 1 -way infinite $D$-Tutte path $P$ from $w_{1}$ and through $w_{r}$.

Since $G$ is 3-indivisible and $H$ is infinite, $J_{1}^{*}$ must be 2 -indivisible. Let $X$ be a path in $J_{1}$ from $w_{1}$ to $V\left(v C q_{1}-\left\{v, q_{1}\right\}\right)$ such that $X \cap C$ consists of a single vertex $x$. Let $J_{1}^{v}$ and $J_{1}^{q}$ denote the subgraphs of $J_{1}^{*}$ such that $v \in V\left(J_{1}^{v}\right), q_{1} \in V\left(J_{1}^{q}\right), J_{1}^{v} \cap J_{1}^{q}=X$, and $J_{1}^{v} \cup J_{1}^{q}=J_{1}^{*}$. Then either $J_{1}^{v}$ or $J_{1}^{q}$ is finite.

Suppose $J_{1}^{q}$ is finite. Then $J_{1}^{q}$ contains a path $Q$ from $w_{1}$ to $q_{1}$ such that $Q$ is contained in the facial cycle of $G$ which contains $\left\{w_{1}, w_{2}, q_{1}, p_{2}\right\}$. Let $J^{\prime}:=J_{1}^{*}+v w_{1}$ 
be the plane graph in which $C^{\prime}:=\left(Q \cup v C q_{1}\right)+v w_{1}$ is a facial cycle. Since $G$ is 3indivisible and $H$ is infinite, $J^{\prime}-V(Q)$ has a unique infinite component, denoted $J^{\prime \prime}$. Then $v \in V\left(J^{\prime \prime}\right)$; for otherwise, by planarity, the neighbors of $J^{\prime \prime}$, which are furtherest apart on $Q$, form a 2-cut $S$ in $G$ such that the component of $G-S$ containing $J^{\prime \prime}$ has no vertex of $C$, contradicting $(4, C)$-connectivity of $G$. By Theorem $(2.8), J^{\prime}$ contains a 1-way infinite $C^{\prime}$-Tutte path $Y$ from $q_{1}$ such that $v w_{1} \in E(Y)$ and $w_{1} \in V\left(q_{1} Y v\right)$. Hence, $Y-v w_{1}$ consists of a path $P^{\prime}$ from $w_{1}$ to $q_{1}$ and a 1-way infinite path $P^{\prime \prime}$ from $v$ such that $P^{\prime} \cap P^{\prime \prime}=\emptyset$ and $P^{\prime} \cup P^{\prime \prime}$ is a $v C q_{1}$-Tutte subgraph of $J_{1}^{*}$.

When $J_{1}^{v}$ is finite, we may apply the same argument to $J_{1}^{*}+q_{1} w_{1}$ as in the preceding paragraph to show that $J_{1}^{*}$ contains a path $P^{\prime}$ from $w_{1}$ to $v$ and a 1 -way infinite path $P^{\prime \prime}$ from $q_{1}$ such that $P^{\prime} \cap P^{\prime \prime}=\emptyset$ and $P^{\prime} \cup P^{\prime \prime}$ is a $v C q_{1}$-Tutte subgraph of $J_{1}^{*}$.

Next we apply Lemma (2.6) to $K:=G-V\left(J_{1}^{*}-\left\{v, q_{1}, w_{1}\right\}\right), H, q_{1} C v, D, P, v, q_{1}, w_{1}$ (as $K, L, Q, Q^{\prime}, T, p, q, u$, respectively). Note that the conditions of Lemma (2.6) are satisfied. In particular, $w_{1}, w_{r} \in V(P)$ implies that $|V(P) \cap V(D)| \geq 2$. Hence, by Lemma (2.6), there is a path $S$ in $K-V(P)$ between $q_{1}$ and $v$ such that $S \cup P$ is a $q_{1} C v$-Tutte subgraph in $K$ and every $P$-bridge of $H$ containing no edge of $D$ is also an $(S \cup P)$-bridge of $K$.

Because $w_{1}, w_{r} \in V(P)$ and by the same argument as in previous lemma, we may assume that $e \in E(S)$. Let $P^{*}:=P \cup S \cup P^{\prime} \cup P^{\prime \prime}$. Then every $P^{*}$-bridge of $G$ is either an $(S \cup P)$-bridge of $H$ or a $\left(P^{\prime} \cup P^{\prime \prime}\right)$-bridge of $J_{1}$. Hence $P^{*}$ is a 2-way infinite $C$-Tutte path in $G$ through $e$.

\section{Tight partial nets}

Let $G$ be a 2-connected infinite plane graph. A tight partial net in $G$ is a sequence $\left(F_{1}, \ldots, F_{n}\right)$ of vertex disjoint non-dividing cycles in $G$, where $n$ is a positive integer, such that $I\left(F_{1}\right)=F_{1}$ and for each $1 \leq i \leq n-1, I\left(F_{i}\right) \subseteq I\left(F_{i+1}\right)$ and every $\left(I\left(F_{i}\right) \cup F_{i+1}\right)$ bridge of $I\left(F_{i+1}\right)$ has at most one attachment on $F_{i+1}$.

A separation of a graph $G$ is an ordered pair $\left(G_{1}, G_{2}\right)$ of subgraphs of $G$ such that $E\left(G_{i}\right) \neq E(G)$ for $i \in\{1,2\}, E\left(G_{1} \cap G_{2}\right)=\emptyset$, and $G_{1} \cup G_{2}=G$. The following observation will be convenient.

(4.1) Lemma. Let $G$ be a 2-connected infinite plane graph and let $\left(F_{1}, \ldots, F_{n}\right)$ be a tight partial net in $G$. Then

(1) there is a plane embedding of $G$ such that $I\left(F_{n}\right)$ is contained in the closed disc in the plane bounded by $F_{n}$, and

(2) for any distinct $x, y \in V\left(F_{n}\right)$, there is a separation $\left(M_{1}, M_{2}\right)$ of $I\left(F_{n}\right)$ such that $\left|V\left(M_{1} \cap M_{2}\right)\right| \leq 2 n, y F_{n} x \subseteq M_{1}$ and $x F_{n} y \subseteq M_{2}$. 
Proof. Note that $F_{n}$ is a facial cycle of $G-V\left(I\left(F_{n}\right)-V\left(F_{n}\right)\right)$. Therefore, $G-V\left(I\left(F_{n}\right)-\right.$ $V\left(F_{n}\right)$ ) has a plane embedding in which the open disc bounded by $F_{n}$ is a face. Since $I\left(F_{n}\right)$ is a finite plane graph, it has a plane embedding such that $F_{n}$ bounds its infinite face. Hence, by combining the new embedding of $G-V\left(I\left(F_{n}\right)-V\left(F_{n}\right)\right)$ and the new embedding of $I\left(F_{n}\right)$, we see that $G$ has a plane embedding in which $I\left(F_{n}\right)$ is contained in the closed disc in the plane bounded by $F_{n}$. Thus we have (1).

To prove (2), we apply induction on $n$. Clearly, (2) holds when $n=1$. So assume $n \geq 2$. For convenience and by (1), let us assume without loss of generality that $I\left(F_{n}\right)$ is contained in the closed disc bounded by $F_{n}$. Because $\left(F_{1}, \ldots, F_{n}\right)$ is a tight partial net, every $\left(I\left(F_{n-1}\right) \cup F_{n}\right)$-bridge of $I\left(F_{n}\right)$ has at most one attachment on $F_{n}$. Thus, there exist vertices $x^{\prime}, y^{\prime} \in V\left(F_{n-1}\right)$ such that $x$ and $x^{\prime}$ are incident with a common face of $G$ and $y$ and $y^{\prime}$ are incident with a common face of $G$. If $x^{\prime}=y^{\prime}$ then by planarity of $I\left(F_{n}\right)$ we see that $I\left(F_{n}\right)$ has a separation $\left(M_{1}, M_{2}\right)$ such that $V\left(M_{1} \cap M_{2}\right)=\left\{x, x^{\prime}=y^{\prime}, y\right\}$, $y F_{n} x \subseteq M_{1}$, and $x F_{n} y \subseteq M_{2}$. So we may assume that $x^{\prime} \neq y^{\prime}$. Then by induction, $I\left(F_{n-1}\right)$ has a separation $\left(M_{1}^{\prime}, M_{2}^{\prime}\right)$ such that $\left|V\left(M_{1}^{\prime} \cap M_{2}^{\prime}\right)\right| \leq 2(n-1), y^{\prime} F_{n-1} x^{\prime} \subseteq M_{1}^{\prime}$, and $x^{\prime} F_{n-1} y^{\prime} \subseteq M_{2}^{\prime}$. Now by planarity of $I\left(F_{n}\right)$, we see that $I\left(F_{n}\right)$ has a separation $\left(M_{1}, M_{2}\right)$ such that $V\left(M_{1} \cap M_{2}\right)=V\left(M_{1}^{\prime} \cap M_{2}^{\prime}\right) \cup\{x, y\}, y F_{n} x \subseteq M_{1}$, and $x F_{n} y \subseteq M_{2} . \square$

The next result is a reduction lemma which shows that, when there is a certain tight partial net with two non-dividing cycles, an infinite graph can be reduced in a certain way so that the existence of a 2-way infinite Tutte paths is preserved. See Figure 3 for an illustration of the situation described in the lemma and its proof. For a subgraph $H$ of a graph $G$, we use $N_{G}(H)$, or simply $N(H)$, to denote the set of vertices in $V(G)-V(H)$ each of which is adjacent to some vertex of $H$.

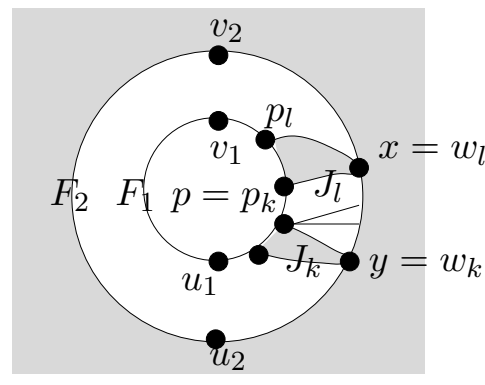

$G$

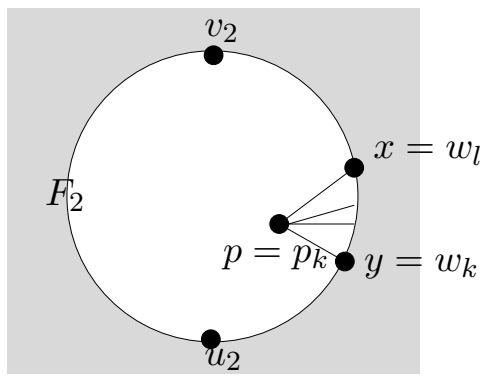

$G^{\prime}$

Figure 3: Illustration for Lemma (4.2)

(4.2) Lemma. Let $G$ be a 2-connected infinite plane graph and $\left(F_{1}, F_{2}\right)$ be a tight partial net in $G$ such that $G$ is $\left(4, F_{1}\right)$-connected. Suppose $I\left(F_{2}\right)$ is contained in the 
closed disc bounded by $F_{2}, v_{i}$ and $u_{i}$ are distinct vertices on $F_{i}$ for $1 \leq i \leq 2$, and $u v \in$ $E\left(v_{1} F_{1} u_{1}\right)$ with $v_{1}, v, u, u_{1}$ on $v_{1} F_{1} u_{1}$ in order. Assume there exist two vertex disjoint paths in $I\left(F_{2}\right)$ from $v_{1} F_{1} v$ to $v_{2} F_{2} u_{2}$ or from $u F_{1} u_{1}$ to $v_{2} F_{2} u_{2}$, which are also internally disjoint from $F_{1} \cup F_{2}$. Then there exist $p \in V\left(v_{1} F_{1} u_{1}-\left\{u_{1}, v_{1}\right\}\right), x, y \in V\left(v_{2} F_{2} u_{2}\right)$, and $f \in\{p x, p y\}$ such that $v_{2}, x, y, u_{2}$ occur on $v_{2} F_{2} u_{2}$ in order and $N\left(x F_{2} y-\{x, y\}\right) \cap$ $V\left(I\left(F_{2}\right)\right) \subseteq\{x, y, p\}$ and such that if $\left(G-V\left(I\left(F_{2}\right)-\left(V\left(F_{2}\right) \cup\{p\}\right)\right)+\{p x, p y\}\right.$ has a 2-way infinite $\left(y F_{2} x+\{p, p x, p y\}\right)$-Tutte path through $f$ then $G$ contains a 2-way infinite $F_{1}$-Tutte path through uv.

Proof. Let $G_{1}$ be the infinite block of $G-V\left(F_{1}\right)$, and let $w_{1}, \ldots, w_{b}$ be the attachments on $G_{1}$ of $\left(G_{1} \cup F_{1}\right)$-bridges of $G$. Because $\left(F_{1}, F_{2}\right)$ is a tight partial net, $F_{2} \subseteq G_{1}$. By planarity, $w_{i} \in V\left(F_{2}\right)$. Without loss of generality, we may assume that $w_{1}, \ldots, w_{b}$ occur on $F_{2}$ in clockwise order. For each $w_{t}, 1 \leq t \leq b$, let $p_{t}, q_{t} \in V\left(F_{1}\right)$ with $p_{t} F_{1} q_{t}$ maximal such that $\left\{p_{t}, w_{t}\right\}$ is contained in a $\left(G_{1} \cup F_{1}\right)$-bridge of $G,\left\{q_{t}, w_{t}\right\}$ is contained in a $\left(G_{1} \cup F_{1}\right)$-bridge of $G$, and any $\left(G_{1} \cup F_{1}\right)$-bridge of $G$ containing some $w_{l} \neq w_{t}$ contains no vertex from $V\left(p_{t} F_{1} q_{t}\right)-\left\{p_{t}, q_{t}\right\}$. See Figure 3. Note that $p_{t}$ and $q_{t}$ are well defined because $G$ is $\left(4, F_{1}\right)$-connected.

We may assume that there are vertex disjoint paths in $I\left(F_{2}\right)$ from $v_{1} F_{1} v$ to $v_{2} F_{2} u_{2}$ and internally disjoint from $F_{1} \cup F_{2}$; the other case can be taken care of in the same way. Then $u v \in E\left(p_{k} F_{1} p_{k+1}\right)$ for some $w_{k} \in V\left(v_{2} F_{2} u_{2}-v_{2}\right)$. We choose such $w_{k}$ that $w_{k} F_{2} u_{2}$ is minimal. Let $J_{k}$ denote the union of $p_{k} F_{1} p_{k+1}$ and those $\left(G_{1} \cup F_{1}\right)$-bridges of $G$ whose attachments are all contained in $V\left(p_{k} F_{1} p_{k+1}\right) \cup\left\{w_{k}\right\}$. Then there is some $w_{r} \in V\left(v_{2} F_{2} w_{k}\right)$ such that $J_{k}-p_{r}$ contains a path from $w_{k}$ to $p_{k+1}$ and through $u v$. Select $w_{r}$ so that $w_{r} F_{2} w_{k}$ is minimal. Let $w_{l}=w_{r}$ if $w_{r} \neq w_{k}$; otherwise, let $w_{l}=w_{k-1}$. Let $J_{l}$ denote the union of $p_{l} F_{1} p_{k}$ and those $\left(G_{1} \cup F_{1}\right)$-bridges of $G$ whose attachments are all contained in $V\left(p_{l} F_{1} p_{k}\right) \cup\left\{w_{l}\right\}$.

Let $G^{\prime}:=G_{1}+\left\{p_{k}, p_{k} w_{j}: w_{j} \in V\left(w_{l} F_{2} w_{k}\right)\right\}$. Since $G$ is $\left(4, F_{1}\right)$-connected, we see that all $\left(G_{1} \cup F_{1}\right)$-bridges of $G$ containing some $w_{j} \in V\left(w_{l} F_{2} w_{k}-\left\{w_{l}, w_{k}\right\}\right)$ are induced by the edge $p_{k} w_{j}$. Hence $G^{\prime}=\left(G-V\left(I\left(F_{2}\right)-\left(V\left(F_{2}\right) \cup\left\{p_{k}\right\}\right)\right)+\left\{p_{k} w_{l}, p_{k} w_{k}\right\}\right.$. Let $F_{2}^{\prime}:=w_{k} F_{2} w_{l}+\left\{p_{k}, p_{k} w_{l}, p_{k} w_{k}\right\}$. See Figure 3 .

Let $f:=p_{k} w_{l}$ and assume that $G^{\prime}$ contains a 2-way infinite $F_{2}^{\prime}$-Tutte path $P^{\prime}$ through $f$. Note that $P^{\prime}-p_{k}$ is an $F_{2}$-Tutte subgraph of $G_{1}$ and $P^{\prime}-p_{k}$ consists of two disjoint 1-way infinite paths. We shall show that the assertion of the lemma holds for $p:=p_{k}$, $x:=w_{l}$ and $y:=w_{k}$.

First, we find a path $S$ from $p_{k+1}$ to $p_{l}$ by applying Lemma (2.6) to $K:=$ $G-V\left(\left(J_{k} \cup J_{l}\right)-\left\{p_{l}, p_{k+1}, w_{l}, w_{k}\right\}\right)$ (with $K, G_{1}, p_{k+1} F_{1} p_{l}, F_{2}, P^{\prime}-p_{k}, p_{k+1}, p_{l}, w_{l}$ as $K, L, Q, Q^{\prime}, T, p, q, u$, respectively). Clearly, the conditions of Lemma (2.6) hold. In particular, we note that $\left|V\left(P^{\prime}-p_{k}\right) \cap V\left(F_{2}\right)\right| \geq 2$. Hence by Lemma (2.6), there is a path $S$ from $p_{k+1}$ to $p_{l}$ in $K-V\left(P^{\prime}-p_{k}\right)$ such that $\left(P^{\prime}-p_{k}\right) \cup S$ is a $p_{k+1} F_{1} p_{l}$-Tutte subgraph of $K$, and every $\left(P^{\prime}-p_{k}\right)$-bridge of $G_{1}$ not containing an edge of $F_{2}$ is a $\left(\left(P^{\prime}-p_{k}\right) \cup S\right)$-bridge 
of $K$.

Let $w_{j}$ denote the endvertex of $P^{\prime}-p_{k}$ other than $w_{l}$. Since $P^{\prime}$ is a 2 -way infinite $F_{2}^{\prime}$ Tutte path in $G^{\prime}$, we see that $w_{k} \in V\left(P^{\prime}\right)$ (for otherwise, the $P^{\prime}$-bridge of $G^{\prime}$ containing $w_{k}$ would have three attachments, namely, $p=p_{k}$ and two on $F_{2}$ ). We shall complete the desired path in $G$ by finding a path from $w_{j}$ to $p_{k+1}$ and a path from $w_{l}$ to $p_{l}$. We distinguish two cases.

Case 1. $p_{k} \neq p_{l}$.

In $J_{l}+w_{l} p_{k}$ we apply Lemma (2.4) to find a $p_{l} F_{1} p_{k}$-Tutte path $P_{l}^{\prime}$ from $p_{l}$ to $p_{k}$ such that $w_{l} p_{k} \in E\left(P_{l}^{\prime}\right)$, and let $P_{l}:=P_{l}^{\prime}-p_{k}$. If $w_{k}=w_{j}$, then in $J_{k}+w_{k} p_{k+1}$ we apply Lemma $(2.5)$ to find a $p_{k} F_{1} p_{k+1}$-Tutte path $P_{k}$ from $w_{k}$ to $p_{k+1}$ such that $p_{k} \in V\left(P_{k}\right)$ and $u v \in E\left(P_{k}\right)$. If $w_{k} \neq w_{j}$, then in $J_{k}+\left\{w_{k} p_{k}, w_{k} p_{k+1}\right\}$ we apply Lemma (2.5) to find a $p_{k} F_{1} p_{k+1}$-Tutte path $P_{k}^{\prime}$ from $w_{k}$ to $p_{k+1}$ such that $w_{k} p_{k}$, uv $\in E\left(P_{k}^{\prime}\right)$; let $P_{k}:=\left(P_{k}^{\prime}-w_{k}\right)+\left\{w_{j}, p_{k} w_{j}\right\}$.

Let $P:=\left(P^{\prime}-p_{k}\right) \cup S \cup P_{k} \cup P_{l}$. Then every $P$-bridge of $G$ is one of the following: a $\left(\left(P^{\prime}-p_{k}\right) \cup S\right)$-bridge of $K$, or a $P_{k}$-bridge of $J_{k}+w_{k} p_{k+1}$ when $w_{k}=w_{j}$, or a $P_{k}^{\prime}$-bridge of $J_{k}+\left\{w_{k} p_{k}, w_{k} p_{k+1}\right\}$ when $w_{k} \neq w_{j}$, or a $P_{l}^{\prime}$-bridge of $J_{l}+w_{l} p_{k}$, or a $P^{\prime}$-bridge of $G^{\prime}$ containing some $w_{i} \in V\left(w_{l} F_{2} w_{j}-\left\{w_{l}, w_{j}\right\}\right)$ (which has three attachments: $p_{k}$, and two on $\left.w_{l} F_{2} w_{j}\right)$. It is easy to see that $P$ gives the desired 2 -way infinite $F_{1}$-Tutte path in $G$ through $u v$.

Case 2. $p_{k}=p_{l}$.

Then $w_{l}=w_{k-1}, w_{j}=w_{k}, J_{l}$ is induced by the edge $w_{l} p_{l}$, and $J_{k}-p_{k}=J_{k}-p_{l}$. Since $J_{k}-p_{l}$ has a path $R$ from $w_{k}$ to $p_{k+1}$ and through $u v$, we let $J_{k}^{\prime}$ denote the union of blocks of $J_{k}-p_{k}$ each of which contains an edge of $R$. Let $R^{\prime}$ denote the path from $w_{k}$ to $p_{k+1}$ containing $u v$ such that $R^{\prime}$ is on the boundary of the face of $G-p_{k}$ which is not a face of $G$. Let $p^{\prime} \in V\left(F_{1} \cap J_{k}^{\prime}\right)$ with $p_{k} F_{1} p^{\prime}$ minimal. By applying Lemma (2.5) we find a $R^{\prime}$-Tutte path $P_{k}$ in $J_{k}^{\prime}$ from $w_{k}$ to $p_{k+1}$ such that $p^{\prime} \in V\left(P_{k}\right)$ and $u v \in E\left(P_{k}\right)$. Let $P:=\left(\left(P^{\prime}-p_{k}\right) \cup S \cup P_{k}\right)+w_{l} p_{l}$. Then every $P$-bridge of $G$ is one of the following: a $\left(\left(P^{\prime}-p_{k}\right) \cup S\right)$-bridge of $K$, or a $P_{k}$-bridge of $J_{k}^{\prime}$, or a $\left(J_{k}^{\prime} \cup\left\{p_{k}\right\}\right)$-bridge of $J_{k}$ with attachments $p_{k}$ and $p^{\prime}$, or a subgraph of $J_{k}$ obtained from a $P_{k}$-bridge $B$ of $J_{k}^{\prime}$ with two attachments by adding $p_{k}$ and all edges from $p_{k}$ to $B-V\left(P_{k}\right)$. Thus, $P$ gives the desired 2-way infinite $F_{1}$-Tutte path in $G$ through $u v$.

The next lemma shows that certain tight partial nets can force the existence of a 2 -way infinite Tutte path. Let $G$ be a 2-connected infinite plane graph which is 3indivisible but not 2-indivisible, let $\left(F_{1}, \ldots, F_{n}\right)$ be a tight partial net in $G$ such that $G$ is $\left(4, F_{1}\right)$-connected, and assume that $I\left(F_{n}\right)$ is drawn in the closed disc bounded by $F_{n}$. For $1 \leq i \leq n$, let $u_{i}, v_{i}$ be distinct vertices of $F_{i}$ such that any two consecutive vertices from $u_{n}, \ldots, u_{1}, v_{1}, \ldots, v_{n}$ are contained in a facial cycle of $G$, and assume that there is no separation $\left(H_{1}, H_{2}\right)$ of $I\left(F_{n}\right)$ such that $\left|V\left(H_{1} \cap H_{2}\right)\right|<2 n,\left\{u_{n}, v_{n}\right\} \subseteq V\left(H_{1} \cap H_{2}\right)$, $v_{n} F_{n} u_{n} \subseteq H_{1}$, and $u_{n} F_{n} v_{n} \subseteq H_{2}$. 
(4.3) Lemma. Let $G,\left(F_{1}, \ldots, F_{n}\right)$, and $u_{i}, v_{i}, 1 \leq i \leq n$, be defined as above. Suppose either (1) $\left(G-V\left(I\left(F_{n}\right)-V\left(F_{n}\right)\right)\right)-\left\{u_{n}, v_{n}\right\}$ has two infinite components or (2) $G-$ $V\left(I\left(F_{n}\right)\right)$ has two infinite blocks, say $H$ and $H^{\prime}$, such that the face of $H$ containing $I\left(F_{n}\right)$ contains $H^{\prime}$ and is bounded by a cycle, and such that no path in $G$ from $H^{\prime}$ to $v_{n} F_{n} u_{n}-\left\{v_{n}, u_{n}\right\}$ is internally disjoint from $I\left(F_{n}\right) \cup H$. Let $u v \in E\left(v_{1} F_{1} u_{1}\right)$ such that $v_{1}, v, u, u_{1}$ occur on $v_{1} F_{1} u_{1}$ in order, and assume when $n \geq 2$ there exist two vertex disjoint paths in $I\left(F_{2}\right)$ from $v_{1} F_{1} v$ to $v_{2} F_{2} u_{2}$ or from $u_{1} F_{1} u$ to $v_{2} F_{2} u_{2}$, which are also internally disjoint from $F_{1} \cup F_{2}$. Then there is a 2-way infinite $F_{1}$-Tutte path in $G$ through $u v$.

Proof. We apply induction on $n$. Suppose $n=1$. If $G-\left\{u_{1}, v_{1}\right\}$ has two infinite components, then by Lemma (3.1) there is a 2-way infinite $F_{1}$-Tutte path in $G$ through $u v$. So assume that $G-V\left(I\left(F_{1}\right)\right)$ has two infinite blocks $H$ and $H^{\prime}$ such that the face of $H$ containing $I\left(F_{1}\right)$ contains $H^{\prime}$ and is bounded by a cycle $D$, and such that no path in $G$ from $H^{\prime}$ to $v_{1} F_{1} u_{1}-\left\{v_{1}, u_{1}\right\}$ is internally disjoint from $H \cup I\left(F_{1}\right)$. Then we see that every $\left(H \cup F_{1}\right.$ )-bridge of $G$ has at most one attachment on $H$ (which must be on $D$ ), and $H^{\prime}$ is contained in an infinite $\left(H \cup F_{1}\right)$-bridge of $G$. Let $w_{1}, \ldots, w_{b}$ denote the attachments on $H$ of $\left(H \cup F_{1}\right)$-bridges of $G$ and let them occur on $D$ in clockwise order. Let $p_{j}, q_{j} \in V\left(F_{1}\right)$ with $p_{j} F_{1} q_{j}$ maximal such that $\left\{p_{j}, w_{j}\right\}$ is contained in an $\left(H \cup F_{1}\right)$-bridge of $G,\left\{q_{j}, w_{j}\right\}$ is contained in an $\left(H \cup F_{1}\right)$-bridge of $G$, and any $\left(H \cup F_{1}\right)$-bridge of $G$ containing some $w_{l} \neq w_{j}$ contains no vertex from $V\left(p_{j} F_{1} q_{j}\right)-\left\{p_{j}, q_{j}\right\}$. Because $G$ is $\left(4, F_{1}\right)$-connected, $p_{j}$ and $q_{j}$ are well defined. Let $J_{j}$ denote the union of $p_{j} F_{1} q_{j}$ and those $\left(H \cup F_{1}\right)$-bridges of $G$ whose attachments are all contained in $V\left(p_{j} F_{1} q_{j}\right) \cup\left\{w_{j}\right\}$. Let $L_{j}$ denote the union of $q_{j} F_{1} p_{j+1}$ and those $\left(H \cup F_{1}\right)$-bridges of $G$ whose attachments are all contained in $V\left(q_{j} F_{1} p_{j+1}\right)$, where $p_{b+1}=p_{1}$. Then there is some $1 \leq j \leq b$ such that $H^{\prime} \subseteq J_{j}$ or $H^{\prime} \subseteq L_{j}$. Recall the assumption that no path in $G$ from $H^{\prime}$ to $v_{1} F_{1} u_{1}-\left\{v_{1}, u_{1}\right\}$ is internally disjoint from $H \cup I\left(F_{1}\right)$. Thus, if $H^{\prime} \subseteq L_{j}$ then $L_{j} \cap F_{1} \subseteq u_{1} F_{1} v_{1}$, whence $u v \notin E\left(L_{j}\right)$; if $H^{\prime} \subseteq J_{j}$ then $u v$ is not in the unique infinite block of $J_{j}-w_{j}$. Hence by Lemma (3.2) and Lemma (3.3), $G$ contains a 2 -way infinite $F_{1}$-Tutte path $P$ through $u v$.

So assume $n \geq 2$. Note that the conditions of Lemma (4.2) are satisfied. By Lemma (4.2), there exist $p \in V\left(v_{1} F_{1} u_{1}-\left\{u_{1}, v_{1}\right\}\right), x, y \in V\left(v_{2} F_{2} u_{2}\right)$, and an edge $f \in\{p x, p y\}$ such that if $G^{\prime}:=\left(G-V\left(I\left(F_{2}\right)-\left(V\left(F_{2}\right) \cup\{p\}\right)\right)+\{p x, p y\}\right.$ has a 2-way infinite $\left(y F_{2} x+\{p, p x, p y\}\right)$-Tutte path through $f$ then $G$ has a 2-way infinite $F_{1}$-Tutte path through $u v$.

Therefore it suffices to show that $G^{\prime}$ has a 2 -way infinite $\left(y F_{2} x+\{p, p x, p y\}\right)$-Tutte path through $f$. For convenience, let $F_{2}^{\prime}:=y F_{2} x+\{p, p x, p y\}$ and assume $f=p x$. There is a tight partial net $\left(F_{2}^{\prime}, \ldots, F_{n}^{\prime}\right)$ in $G^{\prime}$ such that $u_{i} F_{i}^{\prime} v_{i}=u_{i} F_{i} v_{i}$ and $I_{G^{\prime}}\left(F_{i}^{\prime}\right)-\{p x, p y\} \subseteq$ $I_{G}\left(F_{i}\right)$, for all $2 \leq i \leq n$. (This can be shown by applying induction on $i$, by noting that any two consecutive vertices from $u_{n}, \ldots, u_{2}, v_{2}, \ldots, v_{n}$ are contained in a facial cycle of $G^{\prime}$ and that $\left\{u_{i}, v_{i}\right\} \subseteq V\left(I_{G^{\prime}}\left(F_{i}^{\prime}\right)\right)$, and by taking $I_{G^{\prime}}\left(F_{i}^{\prime}\right)$ minimal subject to the 
condition $F_{i}^{\prime} \cap F_{i-1}^{\prime}=\emptyset$.) Note that there is no separation $\left(M_{1}, M_{2}\right)$ of $I_{G^{\prime}}\left(F_{n}^{\prime}\right)$ such that $\left|V\left(M_{1} \cap M_{2}\right)\right|<2(n-1),\left\{u_{n}, v_{n}\right\} \subseteq V\left(M_{1} \cap M_{2}\right), v_{n} F_{n}^{\prime} u_{n} \subseteq M_{1}$, and $u_{n} F_{n}^{\prime} v_{n} \subseteq M_{2}$; for otherwise by planarity $I_{G}\left(F_{n}\right)$ has a separation $\left(H_{1}, H_{2}\right)$ such that $\left|V\left(H_{1} \cap H_{2}\right)\right|<2 n$, $\left\{u_{n}, v_{n}\right\} \subseteq V\left(H_{1} \cap H_{2}\right), v_{n} F_{n} u_{n} \subseteq H_{1}$, and $u_{n} F_{n}^{\prime} v_{n} \subseteq H_{2}$, a contradiction. We claim that when $n \geq 3$ there must be two disjoint paths in $I_{G^{\prime}}\left(F_{3}^{\prime}\right)$ from $v_{2} F_{2}^{\prime} x$ to $v_{3} F_{3}^{\prime} u_{3}$ or from $p F_{2}^{\prime} u_{2}$ to $v_{3} F_{3}^{\prime} u_{3}$, which are internally disjoint from $F_{2}^{\prime} \cup F_{3}^{\prime}$. For otherwise, there exist vertices $u_{2}^{\prime}, v_{2}^{\prime}$ such that all paths in $I_{G^{\prime}}\left(F_{3}^{\prime}\right)$ from $v_{2} F_{2}^{\prime} u_{2}$ to $v_{3} F_{3}^{\prime} u_{3}$ internally disjoint from $F_{2}^{\prime} \cup F_{3}^{\prime}$ intersect $\left\{u_{2}^{\prime}, v_{2}^{\prime}\right\}$. Then $I_{G}\left(F_{n}\right)$ has a separation $\left(H_{1}, H_{2}\right)$ such that $H_{1} \cap H_{2}=\left\{u_{n}, \ldots, u_{3}, u_{2}^{\prime}, v_{2}^{\prime}, v_{3}, \ldots, v_{n}\right\}, v_{n} F_{n} u_{n} \subseteq H_{1}$, and $u_{n} F_{n}^{\prime} v_{n} \subseteq H_{2}$, a contradiction. Therefore, by induction, $G^{\prime}$ has a 2 -way infinite $F_{2}^{\prime}$-Tutte path through $f$.

\section{$5 \quad$ Graphs with $\gamma(G)=0$}

In this section we prove Theorem (1.1) for graphs with no dividing cycles.

(5.1) Theorem. Let $G$ be a 4-connected 3-indivisible infinite plane graph and assume $\gamma(G)=0$. Then $G$ contains a spanning 2 -way infinite path.

Proof. We may assume that $G$ is not 2-indivisible, for otherwise the assertion of this theorem follows from [8] and [9]. Since $G$ is 4-connected and planar and because $\gamma(G)=0$, it follows from Lemma (2.3) that there is a sequence $\left(D_{1}, D_{2}, \ldots\right)$ of non-dividing cycles in $G$ such that

(1) for each $i \geq 1, I\left(D_{i}\right) \subseteq I\left(D_{i+1}\right)$,

(2) for each $i \geq 1, G$ has no finite $I\left(D_{i}\right)$-bridge,

(3) for each $i \geq 1, D_{i} \cap D_{i+1} \subseteq D_{i+1} \cap D_{i+2}$, and

(4) $\bigcup_{i \geq 1} I\left(D_{i}\right)=G$.

If $D_{i} \cap D_{i+1}=\emptyset$ for all $i \geq 1$, then by $(4),\left(D_{1}, D_{2}, \ldots\right)$ is a radial net in $G$, and hence, $G$ is 2-indivisible, a contradiction. So $D_{k} \cap D_{k+1} \neq \emptyset$ for some positive integer $k$. By (3), $D_{i} \cap D_{i+1} \neq \emptyset$ for all $i \geq k$.

Suppose $D_{i} \cap D_{i+1}$ consists of a single path for all $i \geq k$. Then by planarity and because $G$ is 4-connected, for any positive integer $l, D_{i}-V\left(D_{l}\right), i \geq l+1$, are all nonempty and contained in a single component of $G-V\left(I\left(D_{l}\right)\right)$. By (4), for any finite $X \subseteq V(G), X \subseteq V\left(I\left(D_{l}\right)\right)$ for some positive integer $l$. Therefore, $G-X$ has only one infinite component. This shows that $G$ is 2-indivisible, again, a contradiction.

Thus, for some integer $t \geq k, D_{t} \cap D_{t+1}$ consists of at least two vertex disjoint paths, and hence, $G$ has at least two $I\left(D_{t}\right)$-bridges. Because of (2) and since $G$ is 3 -indivisible, $G$ has exactly two $I\left(D_{t}\right)$-bridges, both infinite. So $D_{t} \cap D_{t+1}$ consists of exactly two vertex disjoint paths. Therefore, by (2) and (3), we have 
(5) for each $i \geq t, D_{i} \cap D_{i+1}$ consists of exactly two vertex disjoint paths and $G$ has exactly two $I\left(D_{i}\right)$-bridges (both infinite).

By (5) and by (3), we see that

(6) $\partial G=\bigcup_{i \geq 1}\left(D_{i} \cap D_{i+1}\right)$ and has exactly two components, each of which is a path, or a 1-way infinite path, or a 2-way infinite path.

Let $N_{1}$ and $N_{2}$ denote the two components of $\partial G$. See Figure 4. Note that $I_{G}\left(D_{t}\right) \cap$ $N_{i} \neq \emptyset$ for $i=1,2$. So there is a separation $\left(G_{1}, G_{2}\right)$ of $G$ such that $\left|V\left(G_{1} \cap G_{2}\right)\right|$ is finite, exactly one vertex of $G_{1} \cap G_{2}$ is on $N_{i}$ for each $1 \leq i \leq 2$, and both $G_{1}$ and $G_{2}$ are infinite. Among all such separations $\left(G_{1}, G_{2}\right)$ of $G$, there is one such that

(7) $\left|V\left(G_{1} \cap G_{2}\right)\right|$ is minimum.

Let $u$ be the unique vertex of $G_{1} \cap G_{2} \cap N_{1}$, and $v$ be the unique vertex of $G_{1} \cap G_{2} \cap N_{2}$.

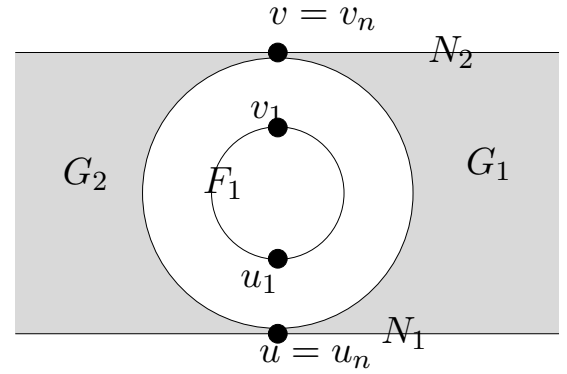

$\left|V\left(G_{1} \cap G_{2}\right)\right|$ is even

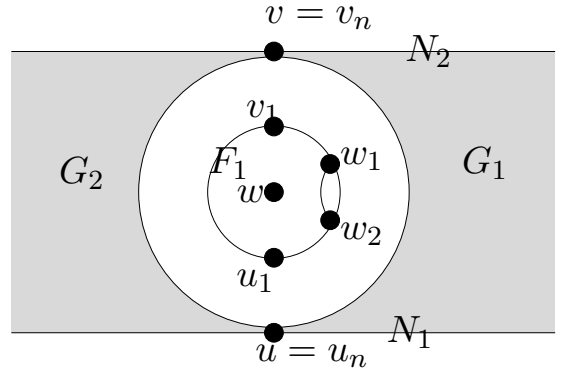

$\left|V\left(G_{1} \cap G_{2}\right)\right|$ is odd

Figure 4: Structure of $G$

When $\left|V\left(G_{1} \cap G_{2}\right)\right|$ is even, let $u_{n}, \ldots, u_{1}, v_{1}, \ldots, v_{n}$ be the vertices in $V\left(G_{1} \cap G_{2}\right)$ such that $u_{n}=u$ and $v_{n}=v$, and any two consecutive vertices from the sequence are contained in a facial cycle of $G$. In this case, let $F_{1}$ denote the facial cycle of $G$ containing $u_{1}$ and $v_{1}$ such that $v_{1} F_{1} u_{1} \subseteq G_{1}$ and $u_{1} F_{1} v_{1} \subseteq G_{2}$. Since $n \geq 2$ (because $G$ is 4-connected), $u_{1}$ and $v_{1}$ each have finite degree and the faces of $G$ incident with $u_{1}$ or $v_{1}$ are bounded by cycles. Hence we may further choose $\left(G_{1}, G_{2}\right)$, subject to $\left\{u_{n}, \ldots, u_{1}, v_{1}, \ldots, v_{n}\right\} \subseteq V\left(G_{1} \cap G_{2}\right)$, so that $v_{1} F_{1} u_{1}$ has at least two edges. (Otherwise, we could simply choose $F_{1}$ to be the other facial cycle of $G$ containing $u_{1} v_{1}$.) Let $H:=G$. See the left part of Figure 4 .

When $\left|V\left(G_{1} \cap G_{2}\right)\right|$ is odd, we let $u_{n}, \ldots, u_{1}, w, v_{1}, \ldots, v_{n}$ be the vertices in $V\left(G_{1} \cap G_{2}\right)$ such that $u_{n}=u$ and $v_{n}=v$, and any two consecutive vertices from the sequence are contained in a facial cycle of $G$. In this case, let $D$ denote the cycle in $G$ such that $I(D)-V(D)=\{w\}$. Because $G$ is 4-connected, $D$ is well defined and $u_{1}, v_{1} \in V(D)$. Without loss of generality, we may assume that $v_{1} D u_{1} \subseteq G_{1}$ and $u_{1} D v_{1} \subseteq G_{2}$. Notice $n \geq 2$ because $G$ is 4 -connected. Hence, $u_{1}, v_{1} \in I\left(D_{i}\right)-V\left(D_{i}\right)$ for all sufficiently large $i$. This implies that $u_{1}$ and $v_{1}$ are of finite degree in $G$ and the faces of $G$ incident with 
$u_{1}$ or $v_{1}$ are bounded by cycles. Hence, we may further choose $\left(G_{1}, G_{2}\right)$ so that, subject to $\left\{u_{n}, \ldots, u_{1}, v_{1}, \ldots, v_{n}\right\} \subseteq V\left(G_{1} \cap G_{2}\right), w$ has at least two neighbors in $v_{1} D u_{1}-$ $\left\{u_{1}, v_{1}\right\}$. (Otherwise, in $V\left(G_{1} \cap G_{2}\right)$ we may replace $w$ with its unique neighbor in $v_{1} D u_{1}-\left\{v_{1}, u_{1}\right\}$, and continue if necessary. This process must stop because of the above finiteness conditions on $u_{1}$ and $v_{1}$.) Therefore, let $w_{1}, w_{2} \in V\left(v_{1} D u_{1}\right)-\left\{v_{1}, u_{1}\right\}$ be distinct neighbors of $w$ such that $v_{1}, w_{1}, w_{2}, u_{1}$ occur on $v_{1} D u_{1}$ in order and $w$ has no neighbor in $V\left(w_{1} D w_{2}\right)-\left\{w_{1}, w_{2}\right\}$. Let $H:=(G-w)+w_{1} w_{2}$. Let $F_{1}=w_{2} D w_{1}+w_{1} w_{2}$, and assume that $w_{1} w_{2}$ is added such that $F_{1}$ is a facial cycle of $H$. See the right part of Figure 4.

Let $\left(F_{1}, \ldots, F_{m}\right)$ be a tight partial net in $H$ such that $m$ is maximum. Note that $m \leq n$. By (1) of Lemma (4.1), we may assume that $I_{H}\left(F_{m}\right)$ is contained in the closed disc bounded by $F_{m}$, as illustrated in Figure 4 .

Suppose $m<n$. If $F_{m} \cap\left(N_{1} \cup N_{2}\right) \neq \emptyset$, then assume by symmetry that $x \in V\left(F_{m} \cap\right.$ $\left.N_{1}\right)$. Then $V\left(F_{m} \cap N_{2}\right)=\emptyset$; for otherwise, let $y \in V\left(F_{m} \cap N_{2}\right)$, then by (2) of Lemma (4.1), $G$ has a separation $\left(H_{1}, H_{2}\right)$ with $\{x, y\} \subseteq V\left(H_{1} \cap H_{2}\right)$ and $\left|V\left(H_{1} \cap H_{2}\right)\right|=2 m$, exactly one vertex of $H_{1} \cap H_{2}$ is on $N_{i}$ for $i=1,2$, and both $H_{1}$ and $H_{2}$ are infinite, contradicting (7). Hence $v_{n} \notin V\left(I\left(F_{m}\right)\right)$. Since $v_{1} \in V\left(I\left(F_{m}\right)\right)$ and every pair of consecutive vertices from $v_{1}, \ldots, v_{n}$ are contained in a facial cycle of $G$, we must have $v_{m} \in V\left(I\left(F_{m}\right)\right)$. Thus there exists some $m \leq j \leq n$ such that $v_{j} \in V\left(I\left(F_{m}\right)\right)$ and $v_{j+1} \notin V\left(I\left(F_{m}\right)\right)$. This shows that $v_{j} \in V\left(F_{m}\right)$ (since $v_{j}$ and $v_{j+1}$ is contained in a facial cycle of $G$ ). By (2) of Lemma (4.1), $I_{H}\left(F_{m}\right)$ has a separation $\left(L_{1}, L_{2}\right)$ such that $\left|V\left(L_{1} \cap L_{2}\right)\right| \leq 2 m$, $\left\{x, v_{j}\right\} \subseteq V\left(L_{1} \cap L_{2}\right), v_{j} F_{m} x \subseteq L_{1}$, and $x F_{m} v_{j} \subseteq L_{2}$. Now it is easy to see that $G$ has a separation $\left(G_{1}, G_{2}\right)$ such that $V\left(G_{1} \cap G_{2}\right)=V\left(L_{1} \cap L_{2}\right) \cup\left\{v_{j+1}, \ldots, v_{n}\right\}$, exactly one vertex of $L_{1} \cap L_{2}$ is on $N_{i}$ for $i=1,2$, and both $G_{1}$ and $G_{2}$ are infinite, contradicting (7). So $F_{m} \cap\left(N_{1} \cup N_{2}\right)=\emptyset$. Therefore $H-V\left(I_{H}\left(F_{m}\right)\right)$ has a unique infinite block $B$ which contains $D_{i}$ for all sufficiently large $i$. Let $F_{m+1}$ denote the cycle bounding the face of $B$ containing $I_{H}\left(F_{m}\right)$. Then we see that $\left(F_{1}, \ldots, F_{m+1}\right)$ is a tight partial net in $H$, which contradicts the choice of $\left(F_{1}, \ldots, F_{m}\right)$.

Hence, $m=n \geq 2$. We may assume that the notation is chosen so that for $1 \leq i \leq n$, $\left\{u_{i}, v_{i}\right\} \subseteq V\left(F_{i}\right), v_{i} F_{i} u_{i} \subseteq G_{1}$, and $u_{i} F_{i} v_{i} \subseteq G_{2}$. Clearly, there is a separation $\left(H_{1}, H_{2}\right)$ of $I_{H}\left(F_{n}\right)$ such that $V\left(H_{1} \cap H_{2}\right)=\left\{u_{1}, \ldots, u_{n}, v_{1}, \ldots, v_{n}\right\}$, and for $1 \leq i \leq n, v_{i} F_{i} u_{i} \subseteq H_{1}$ and $u_{i} F_{i} v_{i} \subseteq H_{2}$. Then by (7) and by planarity, there is no separation $\left(H_{1}, H_{2}\right)$ of $I_{H}\left(F_{n}\right)$ such that $\left|V\left(H_{1} \cap H_{2}\right)\right|<2 n,\left\{u_{n}, v_{n}\right\} \subseteq V\left(H_{1} \cap H_{2}\right), v_{n} F_{n} u_{n} \subseteq H_{1}$, and $u_{n} F_{n} v_{n} \subseteq H_{2}$.

When $\left|V\left(G_{1} \cap G_{2}\right)\right|$ is even, $v_{1} F_{1} u_{1}$ has at least two edges. This, together with $(7)$ and 4-connectivity of $G$, implies that there exist two disjoint paths in $I_{H}\left(F_{2}\right)$ from $v_{1} F_{1} u_{1}-u_{1}$ or from $v_{1} F_{1} u_{1}-v_{1}$ to $v_{2} F_{2} u_{2}$ and internally disjoint from $F_{1} \cup F_{2}$. Hence there is an edge $w_{1} w_{2}$ of $v_{1} F_{1} u_{1}$ such that $v_{1}, w_{1}, w_{2}, u_{1}$ occur on $v_{1} F_{1} u_{1}$ in order and there exist two disjoint paths in $I_{H}\left(F_{2}\right)$ from $v_{1} F_{1} w_{1}$ or from $w_{2} F_{1} u_{1}$ to $v_{2} F_{2} u_{2}$ internally disjoint from $F_{1} \cup F_{2}$. When $\left|V\left(G_{1} \cap G_{2}\right)\right|$ is odd, then by (7), there exist two disjoint paths in $I_{H}\left(F_{2}\right)$ from $v_{1} F_{1} w_{1}$ or from $w_{2} F_{1} u_{1}$ to $v_{2} F_{2} u_{2}$ internally disjoint from $F_{1} \cup F_{2}$. 
Hence by Lemma (4.3), $H$ has a 2 -way infinite $F_{1}$-Tutte path $P$ through $w_{1} w_{2}$. Note that $G$ is 4-connected. Therefore, if $\left|V\left(G_{1} \cap G_{2}\right)\right|$ is even then $P$ is a spanning 2-way infinite path in $G$, and if $\left|V\left(G_{1} \cap G_{2}\right)\right|$ is odd then $\left(P-w_{1} w_{2}\right)+\left\{w, w w_{1}, w w_{2}\right\}$ is a spanning 2-way infinite path in $G$.

\section{Graphs with $\gamma(G)=\infty$}

Let $G$ be a 4-connected 3-indivisible infinite plane graph, and assume $\gamma(G)=\infty$. Let $C$ be a dividing cycle in $G$, let $G^{\prime}$ be the subgraph of $G$ contained in the closed disc of the plane bounded by $C$, and let $G^{\prime \prime}=G-\left(V\left(G^{\prime}\right)-V(C)\right)$. Then $G^{\prime} \cap G^{\prime \prime}=C$, both $G^{\prime}$ and $G^{\prime \prime}$ are 2-indivisible, and both $G^{\prime}$ and $G^{\prime \prime}$ are $(4, C)$-connected.

Let $S^{\prime}$ denote the set of vertices of $G^{\prime}$ of infinite degree. By Theorem (2.1), there is a set $F^{\prime} \subseteq E\left(G^{\prime}\right)$ incident with vertices in $S^{\prime}$ such that $G^{\prime}-F^{\prime}$ has a net $N^{\prime}=$ $\left(C_{1}^{\prime}, C_{2}^{\prime}, \ldots\right)$ satisfying the conclusions of Theorem (2.1) (with $G^{\prime}, S^{\prime}, F^{\prime}, N^{\prime}$ as $G, S, F, N$, respectively). Similarly, let $S^{\prime \prime}$ denote the set of vertices of $G^{\prime \prime}$ of infinite degree. Then by Theorem (2.1), there is a set $F^{\prime \prime} \subseteq E\left(G^{\prime \prime}\right)$ incident with vertices in $S^{\prime \prime}$ such that $G^{\prime \prime}-F^{\prime \prime}$ has a net $N^{\prime \prime}=\left(C_{1}^{\prime \prime}, C_{2}^{\prime \prime}, \ldots\right)$ satisfying the conclusions of Theorem (2.1) (with $G^{\prime \prime}, S^{\prime \prime}, F^{\prime \prime}, N^{\prime \prime}$ as $G, S, F, N$, respectively).

Since $\gamma(G)=\infty, N^{\prime}$ or $N^{\prime \prime}$ must be a radial net. If both $N^{\prime}$ and $N^{\prime \prime}$ are radial nets, then we say that $G$ admits a 2-way radial net. If exactly one of $N^{\prime}$ and $N^{\prime \prime}$ is a radial net, then we slightly abuse notation and say that $G$ admits a mixed net. We deal with these two types of graphs separately.

(6.1) Theorem. Suppose that $G$ is a 4-connected 3-indivisible infinite plane graph, and assume that $G$ admits a 2 -way radial net. Then $G$ contains a spanning 2 -way infinite path.

Proof. Because $G$ has a 2-way radial net, it follows from Lemma (2.2) that

(1) $G$ is locally finite and every face of $G$ is bounded by a cycle.

Let $\left(K^{\prime}, K^{\prime \prime}\right)$ be a separation of $G$ such that $V\left(K^{\prime} \cap K^{\prime \prime}\right)$ is finite, and both $K^{\prime}$ and $K^{\prime \prime}$ are infinite. There exists such a separation $\left(K^{\prime}, K^{\prime \prime}\right)$ of $G$ that

(2) $\left|V\left(K^{\prime} \cap K^{\prime \prime}\right)\right|$ is minimum.

When $\left|V\left(K^{\prime} \cap K^{\prime \prime}\right)\right|$ is even (respectively, odd), then let $u_{n}, \ldots, u_{1}, v_{1}, \ldots, v_{n}$ (respectively, $\left.u_{n}, \ldots, u_{1}, w, v_{1}, \ldots, v_{n}\right)$ be the vertices in $V\left(K^{\prime} \cap K^{\prime \prime}\right)$ such that any two consecutive vertices (in cyclic order) from the sequence are contained in a facial cycle of $G$.

If $\left|V\left(K^{\prime} \cap K^{\prime \prime}\right)\right|$ is even, then let $H:=G$ and let $F_{1}$ be a facial cycle of $H$ containing $\left\{u_{1}, v_{1}\right\}$ such that $v_{1} F_{1} u_{1} \subseteq K^{\prime \prime}$ and $u_{1} F_{1} v_{1} \subseteq K^{\prime}$. Note that $n \geq 2$ (since $G$ is 4connected). Hence, $u_{1}$ and $v_{1}$ have finite degrees in $G$ and faces of $G$ incident with $u_{1}$ 
or $v_{1}$ are bounded by cycles. Hence, we may choose $\left(K^{\prime}, K^{\prime \prime}\right)$ and $F_{1}$ so that $v_{1} F_{1} u_{1}$ has at least two edges.

When $\left|V\left(K^{\prime} \cap K^{\prime \prime}\right)\right|$ is odd, let $D$ be the facial cycle of $G-w$ containing $\left\{u_{1}, v_{1}\right\}$ such that $v_{1} D u_{1} \subseteq K^{\prime \prime}$ and $u_{1} D v_{1} \subseteq K^{\prime}$. Note that $D$ is uniquely defined because $G$ is 4-connected and every face of $G$ is bounded by a cycle (by (1)). Hence we may further select $\left(K^{\prime}, K^{\prime \prime}\right)$ so that, subject to $\left\{u_{1}, \ldots, u_{n}, v_{1}, \ldots, v_{n}\right\} \subseteq V\left(K^{\prime} \cap K^{\prime \prime}\right)$, w has at least two neighbors in $v_{1} D u_{1}-\left\{u_{1}, v_{1}\right\}$. Let $w_{1}, w_{2}$ be distinct neighbors of $w$ in $v_{1} D u_{1}-\left\{u_{1}, v_{1}\right\}$ such that $v_{1}, w_{1}, w_{2}, u_{1}$ occur on $v_{1} D u_{1}$ in order, and $w$ has no neighbor in $w_{1} D w_{2}-\left\{w_{1}, w_{2}\right\}$. Let $H:=(G-w)+w_{1} w_{2}$ and let $F_{1}:=w_{2} D w_{1}+w_{1} w_{2}$. We may assume that if $w_{1} w_{2} \notin E(D)$ then it is represented by a simple arc in the open disc bounded by $D$.

We wish to apply Lemma (4.3); therefore we need to show that

(3) there is a tight partial net $\left(F_{1}, \ldots, F_{n}\right)$ in $H$.

To prove (3), assume that we have a maximum tight partial net $\left(F_{1}, \ldots, F_{k}\right)$ in $H$. For convenience, we may assume that $I_{H}\left(F_{k}\right)$ is contained in the closed disc bounded by $F_{k}$ (see (1) of Lemma (4.1)). Suppose $k<n$.

We claim that $H-V\left(I_{H}\left(F_{k}\right)\right)$ has a unique infinite block. Otherwise, since $H$ is 3-indivisible (because $G$ is), $H-V\left(I_{H}\left(F_{k}\right)\right)$ has a separation $\left(H^{\prime}, H^{\prime \prime}\right)$ such that $\mid V\left(H^{\prime} \cap\right.$ $\left.H^{\prime \prime}\right) \mid \leq 1$ and both $H^{\prime}$ and $H^{\prime \prime}$ are infinite. Since $G$ is 3 -indivisible, $H^{\prime}$ and $H^{\prime \prime}$ each have exactly one infinite component. Let $L^{\prime}$ denote the infinite component of $H^{\prime}$ and $L^{\prime \prime}$ the infinite component of $H^{\prime \prime}$. Since $G$ is 4-connected and $\left|V\left(H^{\prime} \cap H^{\prime \prime}\right)\right| \leq 1, L^{\prime}$ has at least three neighbors on $F_{k}$. Hence by planarity, there are $\{x, y\} \subseteq V\left(F_{k}\right)$ such that $x, y$ are neighbors of $L^{\prime}-V\left(H^{\prime} \cap H^{\prime \prime}\right)$, all neighbors of $L^{\prime}-V\left(H^{\prime} \cap H^{\prime \prime}\right)$ in $F_{k}$ are contained in $x F_{k} y$, and all neighbors of $L^{\prime \prime}-V\left(H^{\prime} \cap H^{\prime \prime}\right)$ in $F_{k}$ are contained in $y F_{k} x$. By (2) of Lemma (4.1), $I_{H}\left(F_{k}\right)$ has a separation $\left(M^{\prime}, M^{\prime \prime}\right)$ such that $\left|V\left(M^{\prime} \cap M^{\prime \prime}\right)\right| \leq 2 k,\{x, y\} \subseteq V\left(M^{\prime} \cap M^{\prime \prime}\right)$, $x F_{k} y \subseteq M^{\prime}$, and $y F_{k} x \subseteq M^{\prime \prime}$. Hence, $H-\left(V\left(M^{\prime} \cap M^{\prime \prime}\right) \cup V\left(H^{\prime} \cap H^{\prime \prime}\right)\right)$ has two infinite components. Therefore, if $\left|V\left(K^{\prime} \cap K^{\prime \prime}\right)\right|$ is even, then $G-\left(V\left(M^{\prime} \cap M^{\prime \prime}\right) \cup V\left(H^{\prime} \cap H^{\prime \prime}\right)\right)$ has two infinite components, and if $\left|V\left(K^{\prime} \cap K^{\prime \prime}\right)\right|$ is odd then $G-\left(V\left(M^{\prime} \cap M^{\prime \prime}\right) \cup V\left(H^{\prime} \cap\right.\right.$ $\left.\left.H^{\prime \prime}\right) \cup\{w\}\right)$ has two infinite components. Since $k<n$ and $\left|V\left(H^{\prime} \cap H^{\prime \prime}\right)\right| \leq 1$, we see that $\left|V\left(M^{\prime} \cap M^{\prime \prime}\right) \cup V\left(H^{\prime} \cap H^{\prime \prime}\right)\right| \leq 2 n-1$. Thus $\left|V\left(M^{\prime} \cap M^{\prime \prime}\right) \cup V\left(H^{\prime} \cap H^{\prime \prime}\right)\right|<\left|V\left(K^{\prime} \cap K^{\prime \prime}\right)\right|$ when $\left|V\left(K^{\prime} \cap K^{\prime \prime}\right)\right|$ is even, and $\left|V\left(M^{\prime} \cap M^{\prime \prime}\right) \cup V\left(H^{\prime} \cap H^{\prime \prime}\right) \cup\{w\}\right|<\left|V\left(K^{\prime} \cap K^{\prime \prime}\right)\right|$ when $\left|V\left(K^{\prime} \cap K^{\prime \prime}\right)\right|$ is odd. This contradicts the minimality of $\left|V\left(K^{\prime} \cap K^{\prime \prime}\right)\right|$ in (2).

Now let $B$ be the unique infinite block of $H-V\left(I_{H}\left(F_{k}\right)\right)$. Because of (1), $H$ is locally finite and every face of $H$ is bounded by a cycle, and only finitely many vertices and edges of $B$ are incident with faces of $H$ which are also incident with vertices of $F_{k}$. Therefore, since $B$ is 2-connected and locally finite, the face of $B$ containing $I_{H}\left(F_{k}\right)$ is bounded by a cycle, say $F_{k+1}$. Because $B$ is the unique infinite block of $H-V\left(I_{H}\left(F_{k}\right)\right)$, $F_{k+1}$ is non-dividing, $I_{H}\left(F_{k}\right) \subseteq I_{H}\left(F_{k+1}\right)$, and every $\left(I_{H}\left(F_{k}\right) \cup F_{k+1}\right)$-bridge of $I_{H}\left(F_{k+1}\right)$ has at most one attachment on $F_{k+1}$. However, this shows that $\left(F_{1}, \ldots, F_{k+1}\right)$ is a tight partial net in $H$, contradicting the choice of $\left(F_{1}, \ldots, F_{k}\right)$. Thus, we have (3). 
By (1) of Lemma (4.1), we may assume $I_{H}\left(F_{n}\right)$ is contained in the closed disc bounded by $F_{n}$. Because $\left\{u_{1}, v_{1}\right\} \subseteq V\left(F_{1}\right)$ and any two consecutive vertices from the sequence $u_{n}, \ldots, u_{1}, v_{1}, \ldots, v_{n}$ are contained in a facial cycle of $H$, we see that $\left\{u_{i}, \ldots, u_{1}, v_{1}, \ldots, v_{i}\right\} \subseteq V\left(I_{H}\left(F_{i}\right)\right)$ for all $1 \leq i \leq n$. Moreover, $\left\{u_{n}, v_{n}\right\} \subseteq V\left(F_{n}\right)$, as otherwise, $F_{n}-\left\{u_{n}, v_{n}\right\}$ is a path or cycle and $G-V\left(K^{\prime} \cap K^{\prime \prime}\right)$ would have only one infinite component (containing $F_{n}-\left\{u_{n}, v_{n}\right\}$ ), a contradiction. Therefore, because $\left\{u_{1}, v_{1}\right\} \subseteq V\left(F_{1}\right), v_{1} F_{1} u_{1} \subseteq K^{\prime \prime}\left(\right.$ or $\left.v_{1} F_{1} u_{1} \subseteq K^{\prime \prime}+w_{1} w_{2}\right)$ and $u_{1} F_{1} v_{1} \subseteq K^{\prime}$, and any two consecutive vertices from $u_{n}, \ldots, u_{1}, v_{1}, \ldots, v_{n}$ are contained in a facial cycle of $H$, it follows from planarity that

(4) for $2 \leq i \leq n,\left\{u_{i}, v_{i}\right\} \subseteq V\left(F_{i}\right), v_{i} F_{i} u_{i} \subseteq K^{\prime \prime}$, and $u_{i} F_{i} v_{i} \subseteq K^{\prime}$.

By (2) and (4), $I_{H}\left(F_{n}\right)$ has no separation $\left(H_{1}, H_{2}\right)$ such that $\left|V\left(H_{1} \cap H_{2}\right)\right|<2 n$, $\left\{u_{n}, v_{n}\right\} \subseteq V\left(H_{1} \cap H_{2}\right), v_{n} F_{n} u_{n} \subseteq H_{1}$, and $u_{n} F_{n} v_{n} \subseteq H_{2}$.

When $\left|V\left(K^{\prime} \cap K^{\prime \prime}\right)\right|$ is even, $v_{1} F_{1} u_{1}$ has at least two edges. This, together with (2) and 4-connectivity of $G$, implies that there exist two disjoint paths in $I_{H}\left(F_{2}\right)$ from $v_{1} F_{1} u_{1}-v_{1}$ or from $v_{1} F_{1} u_{1}-u_{1}$ to $v_{2} F_{2} u_{2}$ internally disjoint from $F_{1} \cup F_{2}$. Hence, there is an edge $w_{1} w_{2}$ of $v_{1} F_{1} u_{1}$ such that $v_{1}, w_{1}, w_{2}, u_{1}$ occur on $v_{1} F_{1} u_{1}$ in order and there are two disjoint paths in $I_{H}\left(F_{2}\right)$ from $v_{1} F_{1} w_{1}$ or from $w_{2} F_{1} u_{1}$ to $v_{2} F_{2} u_{2}$ and internally disjoint from $F_{1} \cup F_{2}$. When $\left|V\left(K^{\prime} \cap K^{\prime \prime}\right)\right|$ is odd, then by (2), there are disjoint paths from $v_{1} F_{2} w_{1}$ or from $w_{2} F_{1} u_{1}$ to $v_{2} F_{2} u_{2}$ and internally disjoint from $F_{1} \cup F_{2}$. Thus by Lemma (4.3),

(5) there is a 2 -way infinite $F_{1}$-Tutte path $P$ through $w_{1} w_{2}$ in $H$.

Now it is easy to see that if $\left|V\left(K^{\prime} \cap K^{\prime \prime}\right)\right|$ is even then $P$ is a spanning 2-way infinite path in $G$, and if $\left|V\left(K^{\prime} \cap K^{\prime \prime}\right)\right|$ is odd then $\left(P-w_{1} w_{2}\right)+\left\{w, w w_{1}, w w_{2}\right\}$ is a spanning 2-way infinite path in $G$.

Finally, we deal with graphs which admit mixed nets.

(6.2) Theorem. Let $G$ be a 4-connected 3-indivisible infinite plane graph, and assume that $G$ admits a mixed net. Then $G$ contains a spanning 2-way infinite path.

Proof. Let $C$ be a dividing cycle in $G$, let $G^{\prime}$ denote the subgraph of $G$ contained in the closed disc bounded by $C$, and let $G^{\prime \prime}:=G-\left(V\left(G^{\prime}\right)-V(C)\right)$. Because $G$ admits a mixed net, we may assume that $G^{\prime}$ has a radial net $N^{\prime}:=\left(C_{1}^{\prime}, C_{2}^{\prime}, \ldots\right)$ with $C \subseteq I_{G^{\prime}}\left(C_{i}^{\prime}\right)$, and for some (possibly empty) set $F^{\prime \prime}$ of edges of $G^{\prime \prime}$ incident with vertices of infinite degree in $G^{\prime \prime}, G^{\prime \prime}-F^{\prime \prime}$ has a ladder net $N^{\prime \prime}:=\left(C_{1}^{\prime \prime}, C_{2}^{\prime \prime}, \ldots\right)$ with $C \subseteq I_{G^{\prime \prime}}\left(C_{1}^{\prime \prime}\right)$. Note that $\partial G^{\prime \prime}$ is a path, or a 1-way infinite path, or a 2-way infinite path. Also note that each face of $G$ is either a face of $G^{\prime}$ or a face of $G^{\prime \prime}$. Thus, in view of Lemma (2.2),

(1) all but one face of $G$ are bounded by cycles, and $\partial G^{\prime \prime}$ is precisely the subgraph of $G$ that lies on the boundary of the exceptional face of $G$.

Because the cycles $C_{i}^{\prime \prime}$ are dividing cycles in $G$ and $C_{i}^{\prime \prime} \cap \partial G^{\prime \prime} \neq \emptyset$ for large $i$, there is a separation $\left(K^{\prime}, K^{\prime \prime}\right)$ of $G$ such that $C_{j}^{\prime} \subseteq K^{\prime}$ and $C_{j}^{\prime \prime} \subseteq K^{\prime \prime}$ for sufficiently large $j$, 
$V\left(G_{1} \cap G_{2}\right)=V\left(C_{i}^{\prime \prime}\right)$ if $\mid V\left(C_{i}^{\prime \prime} \cap \partial G^{\prime \prime} \mid \leq 2\right.$, and $V\left(G_{1} \cap G_{2}\right)$ is the vertex set of the path in $C_{i}^{\prime \prime}$ between two vertices of $\partial G^{\prime \prime}$ and internally disjoint from $\partial G^{\prime \prime}$. Hence,

(2) there exists a separation $\left(K^{\prime}, K^{\prime \prime}\right)$ of $G$ such that $V\left(K^{\prime} \cap K^{\prime \prime}\right)$ is finite, $C_{i}^{\prime} \subseteq K^{\prime}$ for all large $i, C_{i}^{\prime \prime}-V\left(\partial G^{\prime \prime}\right) \subseteq K^{\prime \prime}$ for all large $i$, and $1 \leq\left|V\left(K^{\prime} \cap K^{\prime \prime}\right) \cap V\left(\partial G^{\prime \prime}\right)\right| \leq 2$. Moreover, the vertices in $V\left(K^{\prime} \cap K^{\prime \prime}\right)$ can be ordered as $x_{1}, x_{2}, \ldots, x_{k}$ with $x_{1}, x_{k} \in V\left(\partial G^{\prime \prime}\right), x_{i} \neq x_{j}$ except possibly $x_{1}=x_{k}$, and for each $1 \leq i \leq k-1,\left\{x_{i}, x_{i+1}\right\}$ is contained in a facial cycle of $G$.

Note that we include the possibility $1=\left|V\left(K^{\prime} \cap K^{\prime \prime}\right) \cap V\left(\partial G^{\prime \prime}\right)\right|$, because $\partial G^{\prime \prime}$ may be a trivial path. We choose $\left(K^{\prime}, K^{\prime \prime}\right)$ such that, subject to conditions in (2),

(3) $\left|V\left(K^{\prime} \cap K^{\prime \prime}\right)\right|$ is minimum.

When $\left|V\left(K^{\prime} \cap K^{\prime \prime}\right) \cap V\left(\partial G^{\prime \prime}\right)\right|=2$, let $V\left(K^{\prime} \cap K^{\prime \prime}\right) \cap V\left(\partial G^{\prime \prime}\right)=\{x, y\}$, and otherwise, let $x=y$ be the only vertex in $V\left(K^{\prime} \cap K^{\prime \prime}\right) \cap V\left(\partial G^{\prime \prime}\right)$. Note that when $x=y, G-$ $\left(V\left(K^{\prime} \cap K^{\prime \prime}\right)-\{x=y\}\right)$ has two infinite blocks.

If $\left|V\left(K^{\prime} \cap K^{\prime \prime}\right)-\{x, y\}\right|$ is even (respectively, odd), then let $x=u_{m}, u_{m-1}, \ldots, u_{1}, v_{1}$, $\ldots v_{m-1}, v_{m}=y$ (respectively, $x=u_{m}, u_{m-1}, \ldots, u_{1}, w, v_{1}, \ldots v_{m-1}, v_{m}=y$ ) be the vertices in $V\left(K^{\prime} \cap K^{\prime \prime}\right)$, such that any two consecutive vertices from $u_{m}, \ldots, u_{1}, v_{1}, \ldots v_{m}$ (respectively, $u_{m}, \ldots, u_{1}, w, v_{1}, \ldots v_{m}$ ) are contained in a facial cycle of $G$, and $u_{m} C_{j}^{\prime \prime} v_{m} \subseteq$ $C_{j}^{\prime \prime} \cap C_{j+1}^{\prime \prime}$ for all large $j$. See Figure 5 .

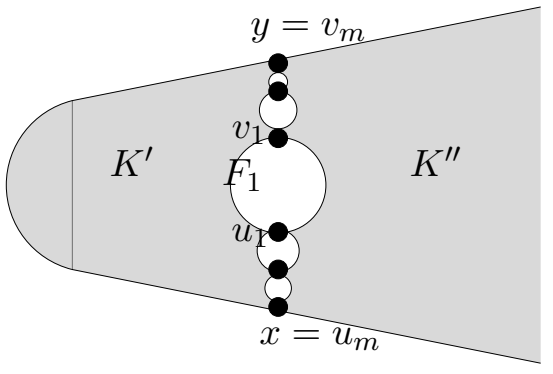

$\left|V\left(K^{\prime} \cap K^{\prime \prime}\right)-\{x, y\}\right|$ is even

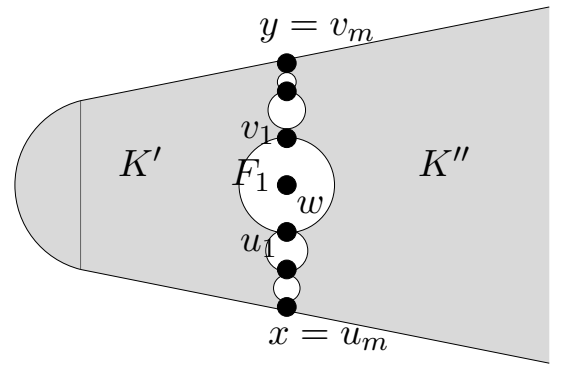

$\left|V\left(K^{\prime} \cap K^{\prime \prime}\right)-\{x, y\}\right|$ is odd

Figure 5: Structure of $G$

When $\left|V\left(K^{\prime} \cap K^{\prime \prime}\right)-\{x, y\}\right|$ is even, let $H:=G$ and let $F_{1}$ be the facial cycle of $G$ containing $u_{1}$ and $v_{1}$ such that $v_{1} F_{1} u_{1} \subseteq K^{\prime \prime}$ and $u_{1} F_{1} v_{1} \subseteq K^{\prime}$. Note that $n \geq 2$, and so, $u_{1}, v_{1} \notin \partial G^{\prime \prime}$. By (1), $u_{1}$ and $v_{1}$ are of finite degree in $G$ and all faces of $G$ incident with $u_{1}$ or $v_{1}$ are bounded by cycles. Hence we may choose $\left(G_{1}, G_{2}\right)$ and $F_{1}$ so that $v_{1} F_{1} u_{1}$ has at least two edges.

When $\left|V\left(K^{\prime} \cap K^{\prime \prime}\right)-\{x, y\}\right|$ is odd, we have $m \geq 2$ (because $G$ is 4-connected). Let $D$ be the facial cycle of $G-w$ containing $u_{1}$ and $v_{1}$ such that $v_{1} D u_{1} \subseteq K^{\prime \prime}$ and $u_{1} D v_{1} \subseteq K^{\prime}$. Because $G$ is 4 -connected and $w \notin \partial G^{\prime \prime}, D$ is well defined and $u_{1}, v_{1} \notin$ 
$V\left(\partial G^{\prime \prime}\right)$. Hence by (1), $u_{1}$ and $v_{1}$ are of finite degree in $G$ and all faces of $G$ incident with $u_{1}$ or $v_{1}$ are bounded by cycles. Therefore, we may further choose $\left(K^{\prime}, K^{\prime \prime}\right)$ so that, subject to $\left\{u_{1}, \ldots, u_{m}, v_{1}, \ldots, v_{m}\right\} \subseteq V\left(K^{\prime} \cap K^{\prime \prime}\right), w$ has two neighbors $w_{1}$ and $w_{2}$ in $v_{1} D u_{1}-\left\{u_{1}, v_{1}\right\}$ such that $v_{1}, w_{1}, w_{2}, u_{1}$ occur on $v_{1} D u_{1}$ in order, and $w$ has no neighbor in $w_{1} D w_{2}-\left\{w_{1}, w_{2}\right\}$. Let $F_{1}:=w_{2} D w_{1}+w_{1} w_{2}$ and $H:=(G-w)+w_{1} w_{2}$. We may assume that if $w_{1} w_{2} \notin E(D)$ then it is represented by a simple arc in the open disc bounded by $D$. Clearly $H$ is $\left(4, F_{1}\right)$-connected. Note that $v_{1} F_{1} u_{1} \subseteq K^{\prime \prime}+w_{1} w_{2}$ and $u_{1} F_{1} v_{1} \subseteq K^{\prime}$.

We wish to apply Lemma (4.3). Let $\left(F_{1}, \ldots, F_{n}\right)$ denote a tight partial net in $H$ such that $n$ is maximum. Then $n \leq m$. By (1) of Lemma (4.1), we may assume that $I_{H}\left(F_{n}\right)$ is contained in the closed disc bounded by $F_{n}$.

Since $u_{1}, v_{1} \in V\left(F_{1}\right)$ and because any two consecutive vertices from $u_{m}, \ldots, u_{1}, v_{1}, \ldots, v_{m}$ or from $u_{m}, \ldots, u_{1}, w, v_{1}, \ldots, v_{m}$ are contained in a facial cycle of $G$, we see that for each $1 \leq i \leq n,\left\{u_{i}, \ldots, u_{1}, v_{1}, \ldots, v_{i}\right\} \subseteq V\left(I_{H}\left(F_{i}\right)\right)$. Therefore, $\left\{u_{n}, v_{n}\right\} \subseteq V\left(F_{n}\right)$, for otherwise, $F_{n}-\left\{u_{n}, v_{n}\right\}$ is a path or a cycle and $H-V\left(I\left(F_{n}\right)\right)$ would have just one infinite component (containing $F_{n}-\left\{u_{n}, v_{n}\right\}$ ). Hence, again because any two consecutive vertices of $u_{m}, \ldots, u_{1}, v_{1}, \ldots, v_{m}$ are contained in a facial cycle of $H$, we see that $u_{i}, v_{i} \in V\left(F_{i}\right)$ for all $1 \leq i \leq n$. By planarity and because $v_{1} F_{1} u_{1} \subseteq K^{\prime \prime}$ (or $v_{1} F_{1} u_{1} \subseteq K^{\prime \prime}+w_{1} w_{2}$ ) and $u_{1} F_{1} v_{1} \subseteq K^{\prime}$, we have $v_{i} F_{i} u_{i} \subseteq K^{\prime \prime}$ and $u_{i} F_{i} v_{i} \subseteq K^{\prime}$ for $2 \leq i \leq n$.

We consider two cases.

Case 1. $n=m$.

Then $H-V\left(I_{H}\left(F_{m}\right)\right.$ ) has two infinite blocks (which is used when applying Lemma (4.3)). Note that $u_{m} \neq v_{m}$, since $H-V\left(I_{H}\left(F_{m-1}\right)\right)$ has a unique infinite block containing $F_{m}$.

Because of the separation $\left(K^{\prime}, K^{\prime \prime}\right),\left(H-V\left(I_{H}\left(F_{m}\right)-V\left(F_{m}\right)\right)\right)-\left\{u_{m}, v_{m}\right\}$ has two infinite components. Hence by planarity and by $(3)$, there is no separation $\left(H^{\prime}, H^{\prime \prime}\right)$ of $I_{H}\left(F_{m}\right)$ such that $\left|V\left(H^{\prime} \cap H^{\prime \prime}\right)\right|<2 m,\left\{u_{m}, v_{m}\right\} \subseteq V\left(H^{\prime} \cap H^{\prime \prime}\right), v_{m} F_{m} u_{m} \subseteq H^{\prime \prime}$, and $u_{m} F_{m} v_{m} \subseteq H^{\prime}$.

When $\left|V\left(K^{\prime} \cap K^{\prime \prime}\right)\right|$ is even, then $m \geq 2$. Since $v_{1} F_{1} u_{1}$ has at least two edges and by (3) and 4-connectivity of $G, I_{H}\left(F_{2}\right)$ has two disjoint paths from $v_{1} F_{1} u_{1}-v_{1}$ or from $v_{1} F_{1} u_{1}-u_{1}$ to $v_{2} F_{2} u_{2}$ internally disjoint from $F_{1} \cup F_{2}$. Hence there is an edge $w_{1} w_{2}$ of $v_{1} F_{1} u_{1}$ such that $v_{1}, w_{1}, w_{2}, u_{1}$ occur on $v_{1} F_{1} u_{1}$ in order and there are two disjoint paths in $I_{H}\left(F_{2}\right)$ from $v_{1} F_{1} w_{1}$ to $v_{2} F_{2} u_{2}$ or from $w_{2} F_{1} u_{1}$ to $v_{2} F_{2} u_{2}$, which are internally disjoint from $F_{1} \cup F_{2}$. When $\left|V\left(K^{\prime} \cap K^{\prime \prime}\right)\right|$ is odd, then by (3), there are two disjoint paths in $I_{H}\left(F_{2}\right)$ from $v_{1} F_{1} w_{1}$ or from $w_{2} F_{1} u_{1}$ to $v_{2} F_{2} u_{2}$, which are internally disjoint from $F_{1} \cup F_{2}$. Hence, the conditions of Lemma (4.3) are satisfied.

By Lemma (4.3), there is a 2-way infinite $F_{1}$-Tutte path $P$ in $H$ through $w_{1} w_{2}$. When $\left|V\left(K^{\prime} \cap K^{\prime \prime}\right)\right|$ is even, we see that $P$ is a spanning 2-way infinite path in $G$. When $\left|V\left(K^{\prime} \cap K^{\prime \prime}\right)\right|$ is odd, then $\left(P-w_{1} w_{2}\right)+\left\{w, w w_{1}, w w_{2}\right\}$ is a spanning 2-way infinite path 
in $G$.

Case 2. $n<m$.

First, we show that $I_{H}\left(F_{n}\right) \cap \partial G^{\prime \prime}=\emptyset$. For otherwise, $F_{n} \cap \partial G^{\prime \prime} \neq \emptyset$. Let $z$ be a vertex contained in $F_{n} \cap \partial G^{\prime \prime}$. Then since $\left(F_{1}, \ldots, F_{n}\right)$ is a tight partial net in $H$, there are vertices $z_{i} \in V\left(F_{i}\right), 1 \leq i \leq n$ such that $z_{n}=z$ and any two consecutive vertices from $z_{n}, \ldots, z_{1}$ are contained in a facial cycle of $H$. Thus, by planarity, either $G-\left\{v_{1}, \ldots, v_{m}, z_{1}, \ldots, z_{n}\right\}$ or $G-\left\{u_{1}, \ldots, u_{m}, z_{1}, \ldots, z_{n}\right\}$ has two infinite components. This contradicts (3) because $n<m$.

Next we show that $H-V\left(I_{H}\left(F_{n}\right)\right)$ has two infinite blocks. For otherwise, assume that $H-V\left(I_{H}\left(F_{n}\right)\right)$ has just one infinite block, say $B$. Because $F_{n} \cap \partial G^{\prime \prime}=\emptyset$, it follows from (1) that all vertices of $F_{n}$ have finite degree in $H$ and each face of $H$ incident with a vertex of $F_{n}$ is bounded by a cycle. Hence the face of $B$ containing $I_{H}\left(F_{n}\right)$ is incident with only finitely many vertices and edges of $H$. Since $B$ is 2-connected, the face of $B$ containing $I_{H}\left(F_{n}\right)$ is bounded by a non-dividing cycle in $H$, denoted $F_{n+1}$. Now it is easy to see that $\left(F_{1}, \ldots, F_{n}, F_{n+1}\right)$ is a tight partial net in $H$, contradicting the choice of $\left(F_{1}, \ldots, F_{n}\right)$.

Let $B^{\prime}, B^{\prime \prime}$ denote the infinite blocks of $H-V\left(I_{H}\left(F_{n}\right)\right)$ such that $C_{j}^{\prime \prime} \subseteq B^{\prime \prime}$ and $C_{j}^{\prime} \subseteq B^{\prime}$ for all sufficiently large $j$. By planarity, we see that the neighbors of $B^{\prime}$ on $F_{n}$ are all contained in $u_{n} F_{n} v_{n}$.

When $\left|V\left(K^{\prime} \cap K^{\prime \prime}\right)\right|$ is even, then $m \geq 2$. Since $v_{1} F_{1} u_{1}$ has at least two edges and by (3) and 4-connectivity of $G, I_{H}\left(F_{2}\right)$ has two disjoint paths from $v_{1} F_{1} u_{1}-v_{1}$ or from $v_{1} F_{1} u_{1}-u_{1}$ to $v_{2} F_{2} u_{2}$ internally disjoint from $F_{1} \cup F_{2}$. Hence there is an edge $w_{1} w_{2}$ of $v_{1} F_{1} u_{1}$ such that $v_{1}, w_{1}, w_{2}, u_{1}$ occur on $v_{1} F_{1} u_{1}$ in order and there are two disjoint paths in $I_{H}\left(F_{2}\right)$ from $v_{1} F_{1} w_{1}$ to $v_{2} F_{2} u_{2}$ or from $w_{2} F_{1} u_{1}$ to $v_{2} F_{2} u_{2}$ which are internally disjoint from $F_{1} \cup F_{2}$. When $\left|V\left(K^{\prime} \cap K^{\prime \prime}\right)\right|$ is odd, then by (3), there exist two disjoint paths in $I_{H}\left(F_{2}\right)$ from $v_{1} F_{1} w_{1}$ or from $w_{2} F_{1} u_{1}$ to $v_{2} F_{2} u_{2}$ which are internally disjoint from $F_{1} \cup F_{2}$. Hence, the conditions of Lemma (4.3) are satisfied.

By Lemma (4.3), we see that $H$ contains a 2 -way infinite $F_{1}$-Tutte path $P$ through $w_{1} w_{2}$. If $\left|V\left(K^{\prime} \cap K^{\prime \prime}\right)-\{x, y\}\right|$ is even, then $P$ is a spanning 2-way infinite path in $G$. If $\left|V\left(K^{\prime} \cap K^{\prime \prime}\right)-\{x, y\}\right|$ is odd, then $\left(P-w_{1} w_{2}\right)+\left\{w, w w_{1}, w w_{2}\right\}$ is a spanning 2-way infinite path in $G$.

It is easy to see that Theorem (1.1) follows from Theorems (5.1), (6.1), and (6.2).

Acknowledgment. The author thanks two referees for their helpful comments. In particular, the author would like to thank the referee who pointed out a missing case in the original proof of Lemma (3.1), brought up references [1] and [5], and helped clarify several ambiguous places. 


\section{References}

[1] R. Halin, Zur häufungspunktfreien Darstellung abzählbarer Graphen in der Ebene, Arch. Math. 17 (1960) 239-243.

[2] C. St. J. A. Nash-Williams, Hamiltonian lines in infinite graphs with few vertices of small valency, Aequationes Math. 7 (1971) 59-81.

[3] C. St. J. A. Nash-Williams, Unexplored and semi-explored territories in graph theory in New Directions in Graph Theory (ed. F. Harary), Academic Press, New York (1973) 149-186.

[4] R. Thomas and X. Yu, 4-Connected projective-planar graphs are hamiltonian, J. Combin. Theory Ser. B 62 (1994) 114-132.

[5] C. Thomassen, Planarity and duality of finite and infinite graphs, J. Combin. Theory Ser. B 29 (1980) 244-271.

[6] C. Thomassen, Infinite graphs in Selected Topics in Graph Theory Vol. 2, Academic Press, New York (1983) 129-160.

[7] C. Thomassen, A theorem on paths in planar graphs, J. Graph Theory, 7 (1983) 169-176.

[8] X. Yu, Infinite paths in planar graphs I, graphs with radial nets, J. Graph Theory 47 (2004) 147-162.

[9] X. Yu, Infinite paths in planar graphs II, structures and ladder nets, J. Graph Theory 48 (2005) 247-266.

[10] X. Yu, Infinite paths in planar graphs III, 1-way infinite paths, J. Graph Theory 51 (2006) 175-198. 